\title{
Molecular allergology and its impact in specific allergy diagnosis and therapy
}

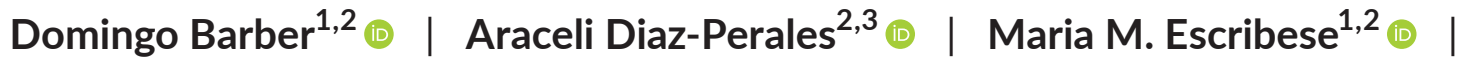

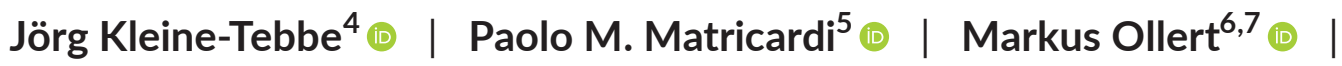 \\ Alexandra F. Santos ${ }^{8,9,10,11}$ (1) | Joaquin Sastre ${ }^{12}$ (1) \\ ${ }^{1}$ Departamento de Ciencias Médicas Básicas, Facultad de Medicina, IMMA, Universidad San Pablo CEU, CEU Universities, Madrid, Spain \\ ${ }^{2}$ ARADyAL-RD16/0006/0015 RD16/0006/0003, Thematic Network and Cooperative Research Centers, ISCIII, Madrid, Spain \\ ${ }^{3}$ Center for Plant Biotechnology and Genomic, Universidad Politécnica de Madrid, Pozuelo de Alarcon, Spain \\ ${ }^{4}$ Allergy and Asthma Center Westend, Berlin, Germany \\ ${ }^{5}$ Department of Pediatric Pneumology and Immunology, Charitè Medical University of Berlin, Berlin, Germany \\ ${ }^{6}$ Department of Infection and Immunity, Luxembourg Institute of Health, Esch-sur-Alzette, Luxembourg \\ ${ }^{7}$ Department of Dermatology and Allergy Centre, Odense University Hospital, Odense, Denmark \\ ${ }^{8}$ Department of Women and Children's Health (Pediatric Allergy, School of Life Course Sciences, Faculty of Life Sciences and Medicine, King's College London, \\ London, UK \\ ${ }^{9}$ Peter Gorer Department of Immunobiology, School of Immunology and Microbial Sciences, King's College London, London, UK \\ ${ }^{10}$ Asthma UK Centre in Allergic Mechanisms of Asthma, London, UK \\ ${ }^{11}$ Children's Allergy Service, Guy's and St Thomas' Hospital, London, UK \\ ${ }^{12}$ Fundación Jiménez Diaz, AllergyDepartment, Universidad Autonomade Madrid, CIBERES, Instituto de Salud Carlos III, Madrid, Spain
}

\section{Correspondence}

Domingo Barber, IMMA, Instituto de Medicina Molecular Aplicada, Facultad de Medicina, Universidad San Pablo CEU, Avda. Monteprincipe s/n, 28668 Boadilla del Monte, Madrid, Spain.

Email: domingo.barberhernandez@ceu.es

\section{Funding information}

This work was supported by Instituto de Salud Carlos III (Project numbers $\mathrm{PI} 19 / 00044$ and PI18/01467). This work was also supported by the grant from Ministerio de Ciencia, Innovación y Universidades RTI2018-095166-B-100.

\begin{abstract}
Progressive knowledge of allergenic structures resulted in a broad availability of allergenic molecules for diagnosis. Component-resolved diagnosis allowed a better understanding of patient sensitization patterns, facilitating allergen immunotherapy decisions. In parallel to the discovery of allergenic molecules, there was a progressive development of a regulation framework that affected both in vitro diagnostics and Allergen Immunotherapy products. With a progressive understanding of underlying mechanisms associated to Allergen immunotherapy and an increasing experience of application of molecular diagnosis in daily life, we focus in analyzing the evidences of the value provided by molecular allergology in daily clinical practice, with a focus on Allergen Immunotherapy decisions.
\end{abstract}

KEYWORDS

allergen immunotherapy, component-resolved diagnosis, food allergy, major allergen, respiratory allergy

Abbreviations: AIT, Allergen Immunotherapy; CRD, Component-resolved diagnosis; EDQM, European Directorate for the Quality of Medicines; EMA, European Medicine Agency; HBV, Honeybee venom; LTP, Lipid transfer protein; PDV, Polistes Dominula venom; POIT, Peanut Oral Immunotherapy; YJV, Yellow Jacket venom. 


\section{1 | INTRODUCTION}

The progressive advance in the knowledge and characterization of allergenic molecules responsible for allergic sensitization to most sources has had a profound impact in the etiological management of allergic disease. This impact can be summarized in three main aspects. Firstly, it has profoundly transformed the way allergenic extracts are characterized and standardized which is legally reflected in the regulatory framework for allergens, especially on the Note for Guidance on Allergenic Extracts, issued by the European Medicine Agency (EMA) and on the allergen monograph of the European Pharmacopoeia, issued by EDQM (Council of Europe), that is adopted by European Directives for Medicinal products. ${ }^{1-3}$ Secondly, it improved the accuracy of allergy diagnosis. Single or multiplexed, allergenic molecules are routinely used and have changed the way we diagnose. ${ }^{4}$ Lastly, but not least, some sensitization profiles are linked to different clinical phenotypes and can be used to stratify allergic patients, to predict intervention outcomes, and to perform system biology studies that open new avenues for allergy disease management. ${ }^{5-11}$

The implementation of a new regulatory framework led to the commercialization of allergen immunotherapy products with registration clinical trials performed during 5 years. Based on these studies, a better understanding of AIT mechanisms is now possible. From allergen-specific effector cell desensitization ${ }^{5,12,13,14}$ to a progressive onset of T- and B-cell-mediated regulatory mechanisms ${ }^{15-19}$ responsible for the disease-modifying effect, their temporal alignment is critical to establish best AIT practices. In this context, the quality of the extract used-and its standardization-the knowledge of patient's sensitization profiles, and the link to the AIT product used is pivotal to maximize the odds of AIT success. In this review, we discuss the different aspects of molecular allergology, from an overview of allergenic molecules and diagnostic methods to the quality of allergen preparation and the clinical approaches to AIT. We aim to offer a practical document to support the allergy specialist in the daily clinical management of allergic patients.

\section{1 | Overview of allergenic molecules}

Currently, more than one thousand allergens from various allergenic sources have been described. Most of these molecules, that are relevant for AIT decisions, belong to a limited number of protein families and have been extensively reviewed. ${ }^{4,20,21}$ More than $40 \%$ of the relevant plant allergens belong to six protein families: $2 \mathrm{~S}$ albumins, non-specific lipid transfer proteins (nsLTP), legumins, vicilins, profilins, and pathogenesis-related (PR)-10 proteins. ${ }^{22}$ In contrast, there are other families with only a few but very relevant members, as they include major pollen allergens such as expansins, polcalcins, pectate lyases, and defensin-like proteins. Concerning animal allergen families, four of them (cysteine proteases, lipocalins, tropomyosins, and parvalbumins) account for over $70 \%$ of the relevant allergens (Table 1). ${ }^{23}$ A separate category is formed by the allergens from hymenoptera venom (Table 2).

\section{Major milestones}

- IgE Discovery (1968)

- First commercial reagents for specific IgE (1973)

- Knowledge and Characterization of the most relevant allergens (1985-2005)

- Development of multiplexed allergens: 1999

- Handbook of Molecular Allergology: 2016

- Progressive use in clinical practice of CRD: 2010-2021

- Progressive understanding of AIT mechanisms: 2010-2021

- First AIT product registered following Pharmaceutical development guidelines: 2007

- First OIT for peanut allergy registered: 2020

\section{Future research perspectives}

- Affinity and avidity need to be explored in IgE response to allergens

- T-cell reactivity to allergens is a critical parameter

- Combination of new biologics and AIT and associated diagnosis will open new intervention strategies

- New regulation of in vitro diagnostics will increase the quality of CRD, but might limit innovation and available molecules

\section{2 | Diagnosis methods and European regulation}

There are different methods available for slgE determinations of single allergenic components. These components might be available as individual diagnostics or multiplexed in arrays. These technologies are commercialized by Thermo Fisher, MADx, Hycor, Euroline, and Siemens. The list of available allergens in each platform has recently been described in detail. ${ }^{20}$ Most of the existing methods for CRD (Component-resolved diagnosis) and multiplexed platforms have been commercialized for research purposes and will need an adaptation and upgrade to comply with new regulatory EU framework as required by the regulation affecting in vitro diagnostics and medical devices, with a transition implementation phase ending by 2025 (REGULATION (EU) 2017/746). This regulation implementation will secure the adequate performance of commercialized diagnostics.

The present document uses as an starting point the handbook of molecular diagnosis by Matricardi and cols that extensively describe best diagnosis strategies combining different approaches. ${ }^{4}$

\section{3 | AIT regulations, biological potency, and link to clinical effect}

The characterization of the main allergenic molecules led to the development of quantification methods and their progressive incorporation into regulations of extract preparation of AIT. In parallel, Create 
TABLE 1 Characteristics and significance of pollen, vegetables, mites, and epithelia allergens available for CRD and relevant for AIT decisions

\begin{tabular}{|c|c|c|c|c|c|}
\hline Source & & Allergen & $\begin{array}{l}\text { Sensitization } \\
\text { rate }\end{array}$ & $\begin{array}{l}\text { Available for } \\
\text { routine CRD }\end{array}$ & Significance \\
\hline \multirow[t]{12}{*}{ Pollen } & \multirow[t]{3}{*}{ Grasses } & Phl p 1 & $>90 \%$ & $S^{*}, M^{*}$ & $\begin{array}{l}\text { Expansin. Present in different plant parts. } \\
\text { Major allergen and usually initiates } \\
\text { sensitization }\end{array}$ \\
\hline & & Phl p 5 & $>60 \%$ & S, M & $\begin{array}{l}\text { Unknown function. Most abundant pollen } \\
\text { protein }\end{array}$ \\
\hline & & Phl p 12 & $5 \%-50 \%$ & S, M & Pan-allergen (food/pollen). Structural protein \\
\hline & Birch & Bet $v 1$ & $>90 \%$ & S, M & $\begin{array}{l}\text { Pan-allergen (food/pollen) } \\
\text { Regulatory protein. Highly expressed in case of } \\
\text { biotic stress. }\end{array}$ \\
\hline & Olive tree & Ole e 1 & $>90 \%$ & S, M & Unknown function \\
\hline & Russian thistle & Sal k 1 & $>90 \%$ & $\mathrm{~S}, \mathrm{M}$ & Pectin methylesterase \\
\hline & Plain tree & Pla a 1 & $>90$ & $\mathrm{~S}, \mathrm{M}$ & Invertase inhibitor \\
\hline & Arizona cedar & Cup a 1 & $>90 \%$ & S, M & $\begin{array}{l}\text { Pectate lyase. Homologous and cross-reactive } \\
\text { to Cry j } 1 \text { from Japanese cedar and Jun a } 1 \\
\text { from mountain cedar }\end{array}$ \\
\hline & English plantain & Pla I 1 & $>70$ & S, M & Ole e 1 like. Non-cross-reactive \\
\hline & Artemisia & Art $v 1$ & $>70$ & S, M & Defensin like \\
\hline & Ragweed & Amb a 1 & & & Pectate lyase \\
\hline & Parietaria & Parj 2 & $>90 \%$ & S, M & ns LTP \\
\hline \multirow{2}{*}{ Foods } & \multirow{2}{*}{ Peanut } & Ara h 8 & & & PR10. Bet v 1 like \\
\hline & & Ara h 9 & & & nsLTP (Pru p 3 related) \\
\hline \multirow[t]{5}{*}{ Mites } & \multirow[t]{4}{*}{$\begin{array}{c}\text { Dermatophagoides } \\
\text { pteronyssinus }\end{array}$} & Der $\mathrm{p} 1$ & $>90 \%$ & S, M & $\begin{array}{l}\text { Cysteine protease. Associated to mite fecal } \\
\text { particles }\end{array}$ \\
\hline & & Der $\mathrm{p} 2$ & $>90 \%$ & S, M & $\begin{array}{l}\text { NCP2. Lipid-binding protein. MD2 link. } \\
\text { Associated to mite bodies }\end{array}$ \\
\hline & & Der $\mathrm{p} 10$ & $<10 \%$ & $\mathrm{~S}, \mathrm{M}$ & Pan-allergen in arthropods. \\
\hline & & Der $\mathrm{p} 23$ & & & Peritrophin-like protein \\
\hline & $\begin{array}{r}\text { Lepidoglyphus } \\
\text { destructor }\end{array}$ & Lep d 2 & $>90 \%$ & M & $\begin{array}{l}\text { Related to Der } \mathrm{p} 2 \text {, but without IgE cross- } \\
\text { reactivity. Cross-reactive with Group } 2 \\
\text { proteins from other minor/storage mites. }\end{array}$ \\
\hline \multirow[t]{4}{*}{ Epithelia } & \multirow[t]{2}{*}{ Cat } & Fel d 1 & $>90 \%$ & $\mathrm{~S}, \mathrm{M}$ & Secretoglobulin. \\
\hline & & Fel d 4,7 & $<50 \%$ & S, M & Lipocalins. Cross-reactivity with Can $\mathrm{f} 1$ \\
\hline & \multirow[t]{2}{*}{ Dog } & Can $\mathrm{f} 1$ & $>50 \%$ & S, M & Lipocalin \\
\hline & & Can $\mathrm{f} 5$ & $>50 \%$ & $\mathrm{~S}, \mathrm{M}$ & $\begin{array}{l}\text { Kalikrein. Serine protease. Regulating semen } \\
\text { liquefaction. Present in male dogs }\end{array}$ \\
\hline
\end{tabular}

Note: Prevalence on food allergens depends on geographical locations and age. In general, storage protein allergy is frequent in pediatric cohorts, while LTP and PR10-linked allergy are of a later onset.

*Estimated from publications. S: Available in singleplex assays. M: Available in Multiplex assays. 
TABLE 2 Characteristics and significance of Hymenoptera venom allergens available for routine CRD (modified according to ${ }^{132}$ ).

\begin{tabular}{|c|c|c|c|}
\hline Allergen & Sensitization rate & $\begin{array}{l}\text { Available for } \\
\text { routine CRD }\end{array}$ & Significance \\
\hline \multicolumn{4}{|l|}{ Honeybee venom (Apis mellifera) } \\
\hline Api $m 3$ Acid phosphatase & $28 \%-63 \%$ & $S^{1}$ & $\begin{array}{l}\text { Marker allergen for HBV sensitization; } \\
\text { Allows discrimination between HBV and YJV/PDV } \\
\text { sensitization; } \\
\text { Valuable marker allergen to diagnose HBV allergy in Api } m \\
\text { 1-negative patients }\end{array}$ \\
\hline Api m 5 Dipeptidyl peptidase IV & $16 \%-70 \%$ & $S^{1}$ & $\begin{array}{l}\text { High cross-reactivity with Ves } v 3 \text { and Pol d } 3 \text { prevents its } \\
\text { use as marker allergen }\end{array}$ \\
\hline Api m 10 Icarapin & $35 \%-73 \%$ & $S^{1,3}, M^{4,5}$ & $\begin{array}{l}\text { Marker allergen for HBV sensitization; } \\
\text { Allows discrimination between HBV and YJV/PDV } \\
\text { sensitization; } \\
\text { Valuable marker allergen to diagnose HBV allergy in Api m } \\
\text { 1-negative patients; } \\
\text { Dominant Api m } 10 \text { sensitization is a putative marker for risk } \\
\quad \text { of VIT failure }\end{array}$ \\
\hline \multicolumn{4}{|l|}{ Yellow jacket venom (Vespula vulgaris) } \\
\hline \multicolumn{4}{|c|}{ European paper wasp venom (Polistesdominula) } \\
\hline Pol d 1 Phospholipase A1 & $87 \%$ & $M^{4}$ & $\begin{array}{l}\text { Marker allergen for PDV sensitization; } \\
\text { Allows discrimination between PDV and HBV sensitization; } \\
\text { High cross-reactivity with Ves } v 1 \text { prevents its use as } \\
\text { marker allergen to discriminate between PDV and YJV } \\
\text { sensitization }\end{array}$ \\
\hline Pol d 5 Antigen 5 & $72 \%$ & $S^{1}, M^{4,5}$ & $\begin{array}{l}\text { Marker allergen for PDV sensitization; } \\
\text { Allows discrimination between PDV and HBV sensitization; } \\
\text { High cross-reactivity with Ves } v 5 \text { prevents its use as } \\
\text { marker allergen to discriminate between PDV and YJV } \\
\text { sensitization }\end{array}$ \\
\hline
\end{tabular}

Abbreviations: CCDs, cross-reactive carbohydrate determinants; CRD, component-resolved diagnostics; HBV, honeybee venom; M, multiplex slgE assay system; PDV, Polistesdominula venom; S, singleplex slgE assay system; YJV, yellow jacket venom.

${ }^{1}$ Thermo Fisher Scientific.

${ }^{2}$ Siemens Healthcare Diagnostics.

${ }^{3}$ Dr. Fooke Laboratories.

${ }^{4}$ Euroimmun.

${ }^{5}$ Macro Array Diagnostics.

${ }^{6}$ Faber test (different suppliers).

${ }^{*}$ Defined by different immunoassays and in different patient populations. Sensitization rates are referenced in the main text.; ${ }^{* *}$ Cross-reactivity with Ves $\vee 2$ and Pol d 2 is possible.; ${ }^{* * *}$ Api m 5 monosensitization may occur in HBV-allergic patients. 
International project resulted in the first available allergen standard csndi Dates and corresponding methods for major allergen content determination. ${ }^{3,24,25,26}$ Today, for any AIT product intended for human use, a proper measurement of biological potency is required and information on major allergen content will become mandatory (a. Bet $v 1$, b. Phl p 5a). Major allergens use to be the most abundant proteins of allergenic sources, and their content in AIT products is closely related to total biological potency. Moreover, most of the patients included in clinical trials are sensitized to major allergens and in a significant percentage monosensitized to them. ${ }^{27}$ In consequence, as a general criterium, patients elected for AIT should be sensitized to major allergens as there is no evidence of the clinical effect of AIT products in patients sensitized only to minor allergens. Unfortunately, the content for minor allergens in AIT preparations is, in the vast majority of cases, unknown and variable. In areas where pollen exposure is very intense, and sensitization to minor allergens is quite frequent, adverse events to AIT preparations containing variable amounts of minor allergens variability have been described. ${ }^{28,29}$

Main AIT mechanisms involve early effector cells desensitization and progressive onset of tolerance through a regulatory response that needs at least 3 years to be consolidated. ${ }^{5,13}$ Dose-finding studies are usually performed in short-term studies, and thus effective dose is adjusted to target preferentially early immunological mechanism. Again, major allergen doses tolerated by patients sensitized to major allergens might serve as basis for dose definition in AIT.

\section{4 | CRD: Monitoring of allergy progression}

Birth cohort studies have shown a sequential broadening of the IgE response to complex allergen sources. Preliminary studies performed so far suggest that IgE response starts with a monomolecular stage and then, through an oligomolecular sensitization pattern (2-4 specificities) becomes polymolecular(>4). ${ }^{30}$ This "molecular spreading" has been observed in many participants in the MAS birth cohort allergic to Timothy grass. In this case, the "initiator molecule" was Phl p 1. However, only a few patients develop an extremely polymolecular response, producing IgE antibodies to all diagnostically available allergenic molecules of Phleum pratense (Phl p 1, Phl p 2, Phl p 4, Ph p 5, Phl p 6, Phl p 7, Phl p 11, and Phl p 12). ${ }^{30}$ Similarly, molecular spreading has been observed in the MAS cohort among children developing IgE sensitization against Dermatophagoidespteronyssinus. ${ }^{31}$ In this case, multiple "initiator molecules" have been observed (Der p 1, Der p 2, and Der p 23). In these children, sensitization started with Der p 1 and/or Der p 2 and/or Der p 23 (defined as group "A" molecules); then involved Der p 4, Der p 5, Der $p$ 7, and Der $p 21$ (group "B" molecules); and was completed with IgE to Der $p$ 11, Der p 14, Der p 15, Der p 18, and clone 16 (group " $C$ " molecules). This expansion of the IgE response has also been defined as "the $A B C$ march" of mite allergy. ${ }^{31}$ Children with a broader polymolecular IgE sensitization pattern were also more frequently affected by asthma when compared with those who remained in the " $A$ " stage of IgE sensitization. ${ }^{31} \mathrm{~A}$ similar trend was observed among grass pollen allergic children in the Manchester Allergy and Asthma Study (MAAS). ${ }^{32}$ In this case, children with broader molecular patterns of IgE sensitization had also a significantly increased risk for asthma, eczema, and rhinitis. ${ }^{32}$ It has also been proposed that earlier administration of AIT, that is, at a mono- or oligomolecular stage of sensitization, may be more effective than at later stages. ${ }^{33}$

\section{I CRD AND SELECTION OF PREPARATIONS FOR AIT}

\section{1 | Grass pollen allergy}

Grasspollen allergy is probably the most studied allergic condition. There is a broad allergen panel for CRD of grass allergy, and a SLIT AIT product that is the only one with a complete clinical development program, including several 5-year studies reviewed by Barber et al, ${ }^{12}$ that provides the best available information to understand the link between sensitization profiles and AIT.

Allergen composition studies of a Phleum pollen extract ${ }^{34}$ allowed to establish that $\mathrm{Phl} \mathrm{p} 5$ and its related allergen $\mathrm{Phl} \mathrm{p} 6$ are the most abundant proteins, accounting for more of $50 \%$ of protein content. Accumulated content of $\mathrm{Phl} \mathrm{p} 1$ represents less than $10 \%$. The rest of allergen components are in a range of $1 \%-10 \%$ of total protein. Despite its relatively low abundance, $\mathrm{Phl}$ p 1 is the most relevant single allergen sensitizer of grass allergic patients. Epidemiological studies performed in Spain ${ }^{35,36}$ and CRD analysis of 1905 patients included in AIT clinical trials of grass pollen allergy in North America ${ }^{27}$ demonstrated the preponderance of $\mathrm{Phl}$ p1 as primary sensitizer to grass pollen. In fact, a significant proportion of patients were mono-sensitized to this allergen. Similar results in pediatric grass pollen allergic patients have been reported. ${ }^{37}$

Recently, a potential explanation for this has been published. ${ }^{38}$ Group 1 grass allergens, belonging to the beta expansins family, are present in other plant parts. In Autumn, upon plant death, plant particles are aerosolized and might be presented together with Alternaria spores, initiating sensitization process. No loss of effect in AIT is observed in patients mono-sensitized to either Phl p 1 or $\mathrm{Phl} \mathrm{p} 5^{27}$; however, patients with low (first tertile) slgE to any of the two major allergens presented no clinical benefit in the first pollen treatment season. Presumably, early AIT effects are governed by desensitization and initial immunological events in a dose-dependent manner. Patients with low slgE levels might need a higher allergen dose to get desensitized; however, in a 5-year mechanistic study, ${ }^{9}$ an impaired regulatory response for this type of patients was not observed, and thus, they should not be excluded for AIT if they meet clinical inclusion criteria.

\section{2 | The role of pan-allergens}

An interesting subgroup of grass pollen allergic patients are sensitized to profilin, a pan-allergen present in all vegetable tissues, with 
a highly conserved structure. Usually, profilin is a confounding diagnostic factor, which is minimized by CRD diagnosis either "in vitro" or even "in vivo". ${ }^{39}$ Profilin sensitization strongly associates with grass pollen allergy ${ }^{35}$ probably due to a relatively high profilin content in grass pollen compared to other pollens. ${ }^{40}$ Usually, sensitized profilin patients present no food-mediated reaction or only local (oral) ones to, that is, sweet melon species. However, in areas of very high grass pollen exposure, some allergic patients do not respond to AIT and present severe food profilin-mediated reactions. ${ }^{41,42}$ In fact, T-cell reactivity to profilin increases along with grass pollen intensity exposure. Moreover, in patients from central Spain, T-cell proliferation induced by profilin is similar to that induced by the major allergen Phl p $1 .^{34}$ These peculiar patients, as well as others exposed to very high pollen levels, can be identified by combining CRD with clinical evaluation and provide invaluable models to understand allergic inflammation and AIT mechanisms and its limitations.

Finally, patients sensitized simultaneously to the pollen panallergens polcalcin and profilin have been shown to have twice as many primary sensitizations and to duplicate the duration of allergic disease, ${ }^{36}$ suggesting that AIT might not be effective. Similar results supporting clinical value for Phlp 7 and Phl p 12 have been described in pediatric cohorts. ${ }^{37}$ Moreover, this specific double sensitization profile to pan-allergens is not linked to any particular pollen. ${ }^{36} \mathrm{At}$ this moment, other grass allergens present a limited diagnostic value for AIT decisions and some of them provide confounding information due to lack of specificity. ${ }^{43}$

Figure 1 represents an algorithm to support decision-making in the selection of AIT for grass pollen allergy.

\subsection{Olive pollen allergy and nsLTP food allergy}

One of the best models to understand the link between pollen exposure, sensitization profiles, and clinical impact is the olive pollen allergy model. Minor allergen sensitization is frequent in areas of very high exposure. Interestingly, sensitization to Ole e 7-the nsLTP from olive pollen-is associated with a unique clinical phenotype. ${ }^{29,35}$ Patients living in areas with high olive pollen exposure and sensitized to Ole e 7 develop a severe respiratory allergic phenotype. These patients do not respond to AIT and constitute another model to understand evolution to severe allergic phenotypes. In Figure 2, an algorithm to support the selection of olive pollen AIT is shown.

There are other pollens where nsLTPs play a preponderant role, as Parietariajudaica, whose main allergen is a LTPs, or Artemisia artimisifolia and Platanus orientalis, whose Art $v 3$ or Plaa 3 is LTPs. Art

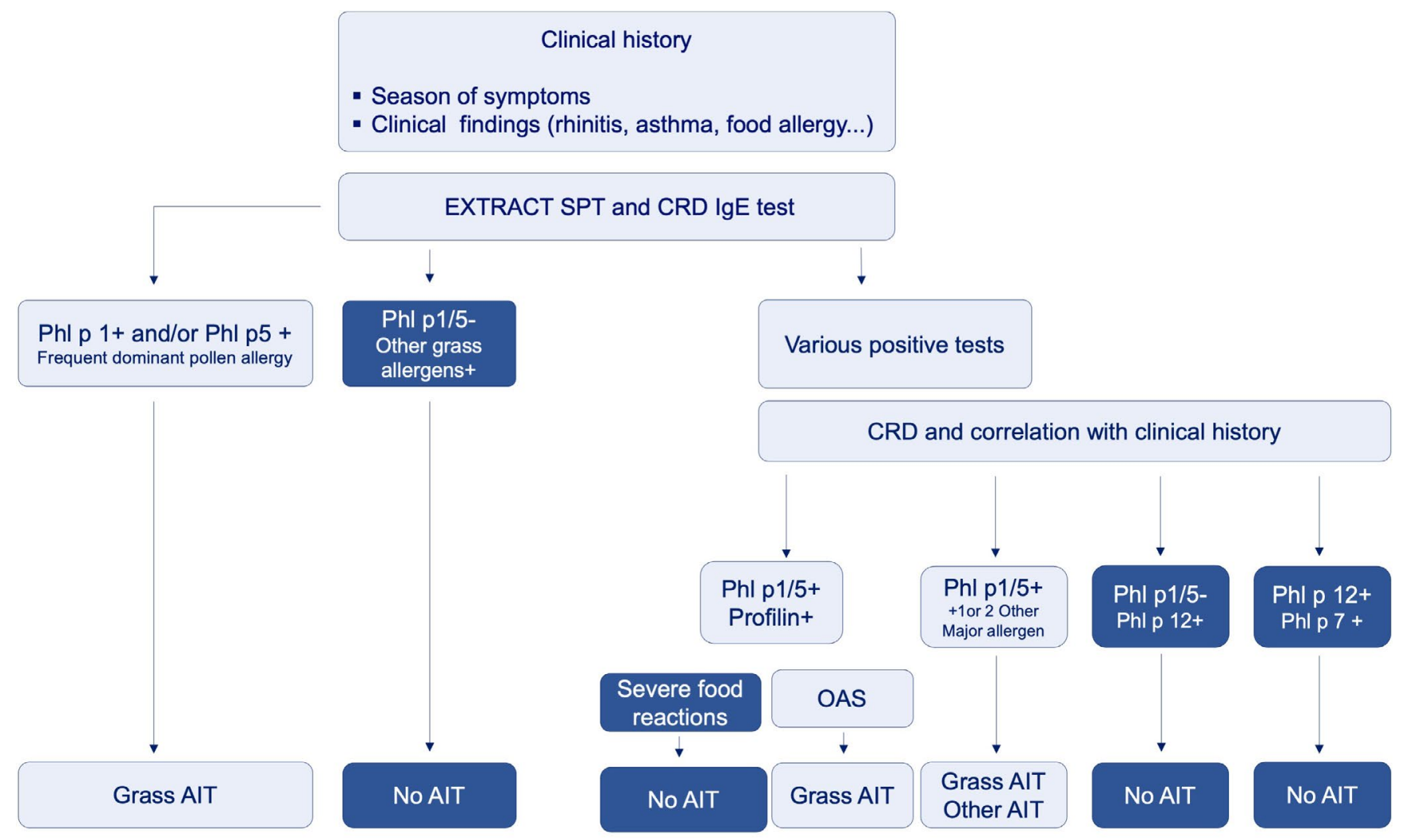

FIGURE 1 Diagnostic algorithm and decision tree for AIT using CRD in Grass pollen allergy. Grass pollen allergy is one of the most studied allergy models. Major allergen sensitization is required before considering AIT. The combination of major allergens and pan-allergens provides the necessary tools for AIT decisions. Profilin allergy might be a contraindication only in severe food allergic patients, while double sensitization to both pan-allergens is associated to many years of disease evolution, poly-sensitization, and poor intervention outcome 


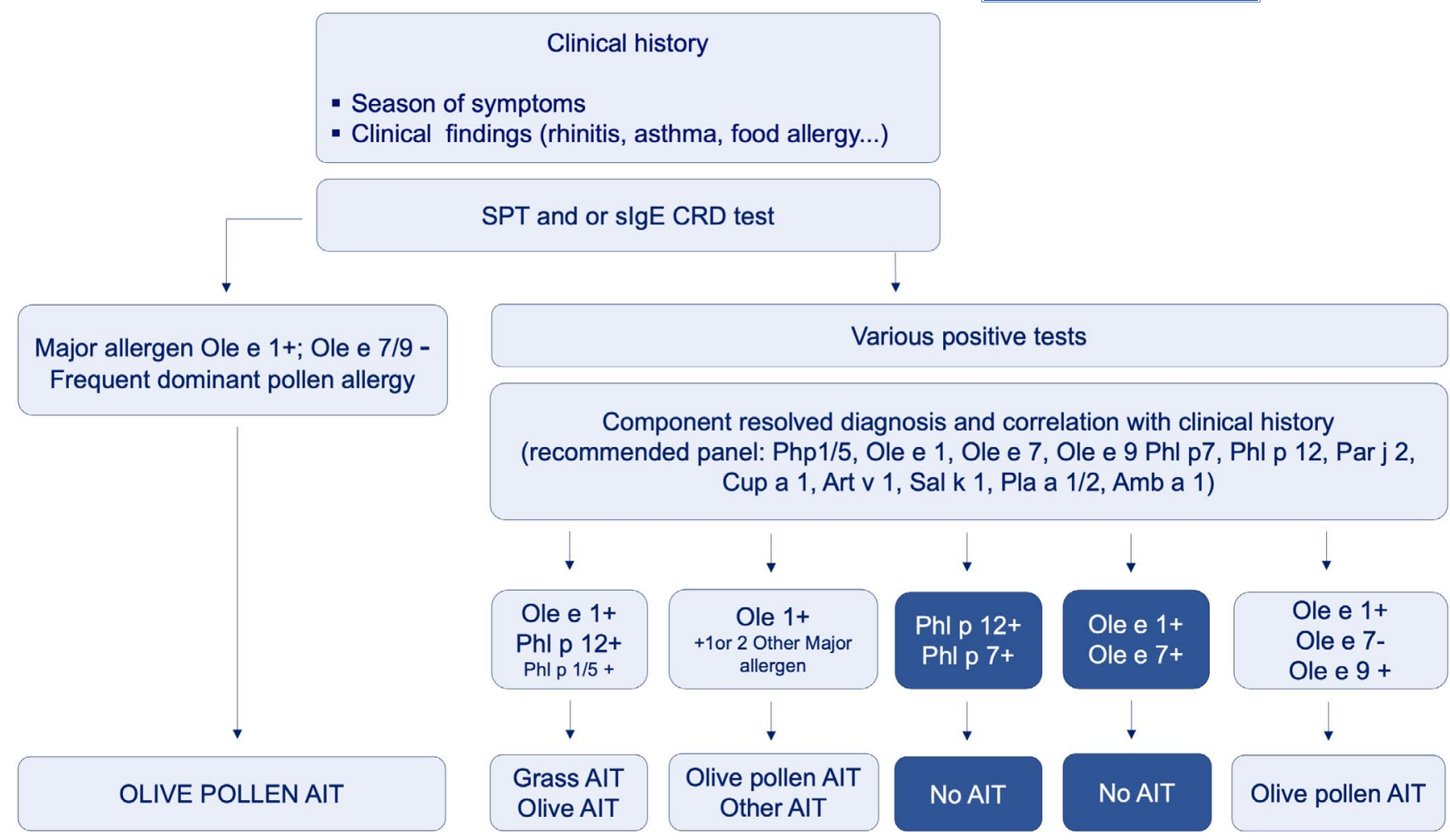

FIGURE 2 Diagnostic algorithm and decision tree for AIT using CRD in Olive pollen allergy. Olive pollen allergies are one of the most complex allergy models. Usually olive cultivars cover homogeneous areas and present acute differences in allergen pollen content. In dense pollen areas, patients are exposed to the highest pollen counts recorded. Th existing of complex profiles, marked by minor allergens sensitization, makes CRD a fundamental tool for patient management and AIT decisions

v 3 and Pla a 3 cross-react with Pru p 3complicating the differential diagnosis of the primary sensitization (to pollen or peach).

LTP-mediated allergy is the predominant food allergy in adults in the Mediterranean Area and Southern European countries. ${ }^{44,45}$ Pru p 3-the peach LTP-is the best marker for LTP allergy. Sublingual AIT has proved to be effective for the treatment of LTP allergy, with clinical effect not only against allergy to closely related food species such as Rosaceae fruits, ${ }^{46}$ but also against allergy to species with about only $60 \%$ sequence identity, such as the Peanut LTP, Ara h $9 .{ }^{47,48}$ Evaluating the spectrum of recognition to multiple LTPs is helpful to make a potential AIT therapeutic decision. Patients sensitized to LTPs distant to Pru p 3 such as wheat Tri a 14 might not benefit completely from AIT based on Pru p 3. Unfortunately, SLIT AIT for LTP allergy is only available in a limited number of Countries. Figure 3 summarizes decision trees for LTP-mediated AIT.

\subsection{Birch and Oak tree pollen allergy and PR10 proteins}

PR10 allergens are present in multiple species of Fagales tree pollen. Among them, birch in Europe ${ }^{49}$ and oak in North America are regarded as primary sensitizers. There are products registered worldwide for the treatment of birch pollen allergy. ${ }^{50}$ Recent data suggest that a birch-based AIT Product within defined Bet v 1 content has clinical benefit during oak pollen season in North America. ${ }^{51}$ Interestingly, a study analyzed the effect of birchbased AIT in cross-reactive T-cell response to homologous PR10 allergens from other trees. ${ }^{52}$ This study demonstrated that downregulation of T-cell reactivity can be achieved against multiple, T-cell cross-reactive PR10 molecules. The control of T-cell proliferation based on T-cell cross-reactivity might be the most relevant endpoint for successful AIT. As in the case of profilins, PR10 proteins are also present in multiple fruit, nut, legume, and vegetables species, leading to PR10-associated allergy symptoms. There is limited evidence on the clinical benefit of PR10-based pollen AIT in the amelioration of pollen-related food allergy syndrome (FPAS). ${ }^{53,54}$ However, immunotherapy with Mal $\mathrm{d} 1$ has been associated with clinical improvement. ${ }^{55}$ More studies will be needed to clarify potential use of PR10 pollen AIT in this particular type of secondary food allergy

\section{5 | Cypress/cedar allergy}

Japanese cedar pollen is the main cause for seasonal respiratory allergy in Japan, and Cupressaceae are relevant allergens in the United States and southern Europe as well. Major allergens belonging to the pectate lyase family (Cup a 1, Cup s 1, Jun a 1 and Cry j1) show a very high cross-reactivity. 


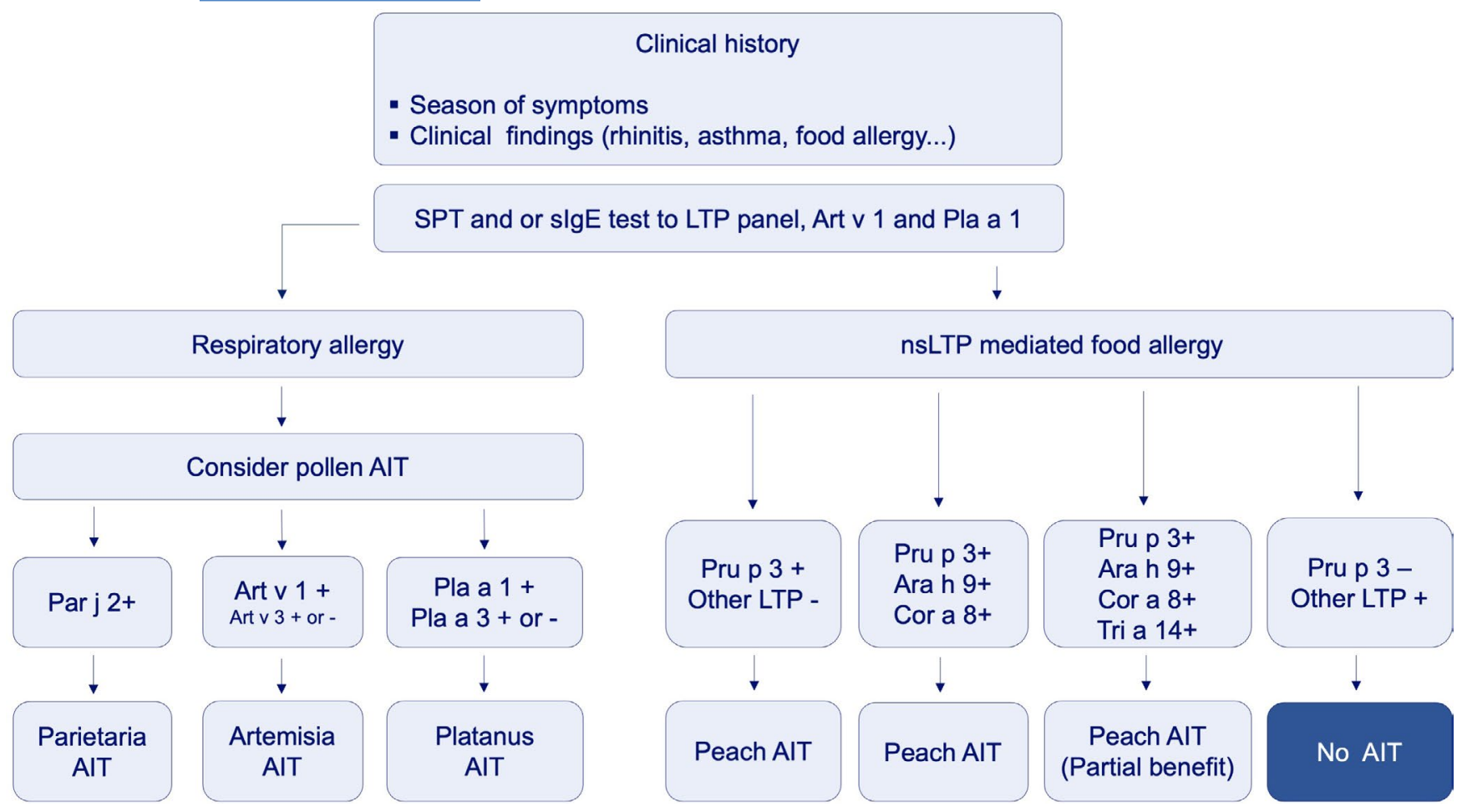

FIGURE 3 Diagnostic algorithm and decision tree for AIT using CRD in nsLTPs-mediated allergies. The existence of cross-reactivity between nsLPTs from Artemisia and Platanus pollens makes CRD necessary. LTP immunotherapy with an enriched Pru p 3 can be considered in Countries with availability of this type of therapy

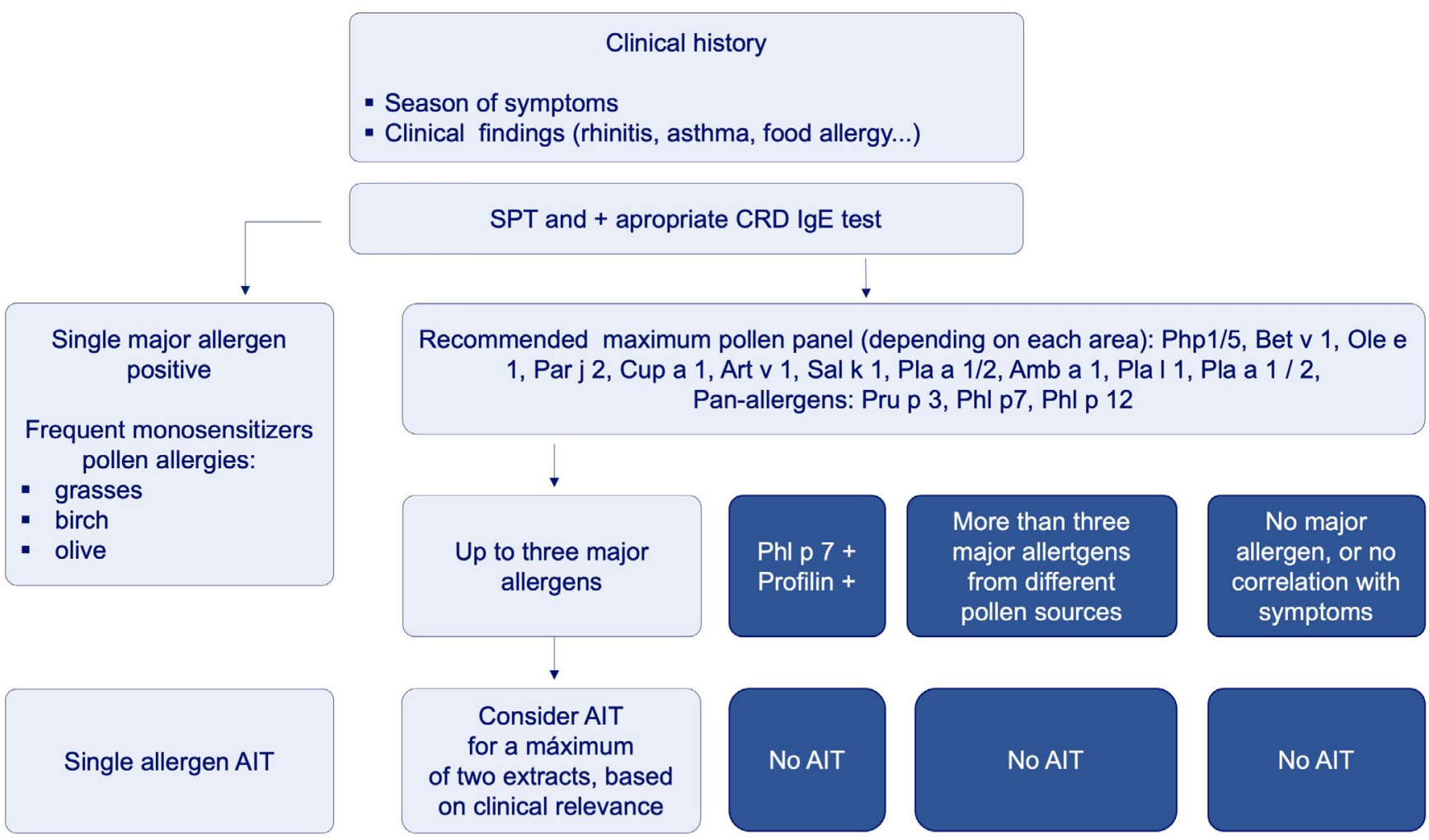

FIGURE 4 Diagnostic algorithm for in vitro diagnostics and Decision tree for AIT using CRD in pollen allergies. Discrimination of primary sensitizers and pan-allergens positivity is needed. AIT guidelines do not recommend AIT for poly-sensitized patients 
There is only one AIT product ${ }^{56,57}$ with clinically documented efficacy. The recent registration of a Japanese cedar AIT product in Japan opens the possibility-as in the case of birch/oak AIT-to explore its value for treating cedar/cypress allergy worldwide.

\section{6 | Ragweed pollen allergy}

Ragweed allergy dominates weed pollen allergy in North America and, after the accidental introduction of ragweed in Europe about 100 years ago, is in continuous progression on the continent.

Major allergen Amb a 1, a pectate lyase, is a true marker of ragweed allergy and presents low cross-reactivity with related proteins from other pollen sources. ${ }^{58}$ However, there is an extensive crossreactivity among different Ambrosia species. ${ }^{59}$ Currently, there are AIT products extensively documented in adults ${ }^{60}$ both in American and European patients as well as in children ${ }^{61}$ and these products are available worldwide for AIT practice.

\subsection{Other pollen allergies}

There is a limited clinical evidence of AIT benefit in the treatment of other pollen allergies. However, data on major allergens can be extrapolated from studies on existing registered pollen AIT products. Major specific allergens available for diagnosis include the following: Art v 1 (Artemisia artimisifolia), Salk k 1 (Salsola Kali), Pla I 1 (Plantago lanceolata), and Pla a 1 (Platanus acerifolia). ${ }^{58}$

Figure 4 represents an algorithm to support decision-making in the selection of AIT in multiple pollen allergies.

\subsection{Cat and dog allergies}

In general, the severity and progression of cat and dog allergies involve IgE recognition of a progressively increasing number of components from the sensitizing allergen source (molecular spreading). ${ }^{30,62,63,64,65}$

Despite the fact that AIT with animal-derived extracts is clinically rarely indicated particularly due to risks in poorly controlled asthmatic patients, the availability of CRD for different cat and dog allergens has also raised the possibility of more precisely targeted interventions. CRD may distinguish primary sensitization from crosssensitization, thereby enabling selection of the primary sensitizing allergen source for therapy.

In a recent, comprehensive study, ${ }^{66}$ including most of the components available, the pattern of IgE sensitization to cat allergens showed that $92 \%$ of cat-allergic patients had positive IgE antibodies results to Fel $d$ 1. Other allergens also seem important, such as Fel $d$ 4 and Fel $d$ 7. Previous studies reported similar results. ${ }^{67}$ The content of Feld d 1 in allergenic extracts varies substantially. Nevertheless, it has been demonstrated that the maintenance efficacy dose of Fel d 1 is $15 \mu \mathrm{g} / \mathrm{ml}^{68}$

In the same study, in patients with dog allergy, $52.4 \%$ were positive to Can $\mathrm{f}$ 1, and $57.2 \%$ to Can $\mathrm{f}$ 5. The most frequent monomolecular sensitization was to Can $\mathrm{f} 5$.

The content of different dog allergen molecules in European AIT extracts has been recently studied. ${ }^{69}$ These authors demonstrated great variability in extracts from five companies and scarce content of major allergens. ${ }^{70}$

Based on limited evidence, AIT with cat extracts yield better clinical results than with dog ones. The higher complexity of dog allergy
FIGURE 5 Diagnostic algorithm for in vitro diagnostics in epithelia allergy and associated decision tree for AIT using CRD. The proposed algorithm discriminates between primary sensitization and cross-reactive slgE response. Only patients sensitized to major allergens should be eligible for AIT
Clinical history

- Correlation of nasal and bronchial symptoms with exposure to pet - Other sensitisation

\section{EXTRACT SPT + and CRD IgE test}

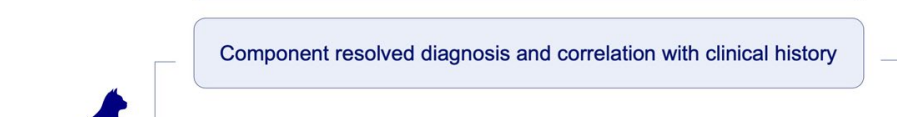

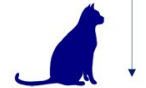

Feld $1+$ Fel d $2 / 4$ Frequent dominant cat allergy

Fel d $1+$

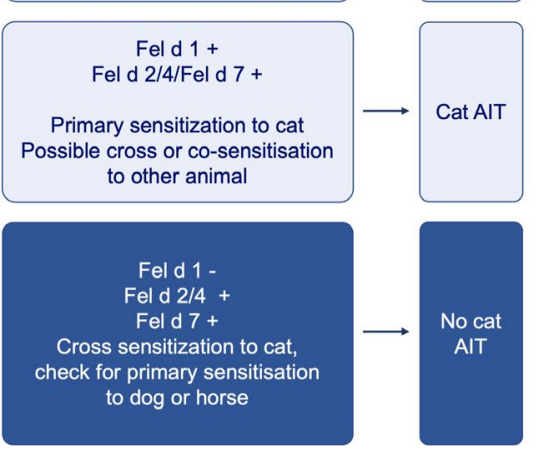

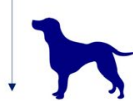

\section{Can $1 / 2 / 5+$} Can $f$ 3Primary sensitisation to dog
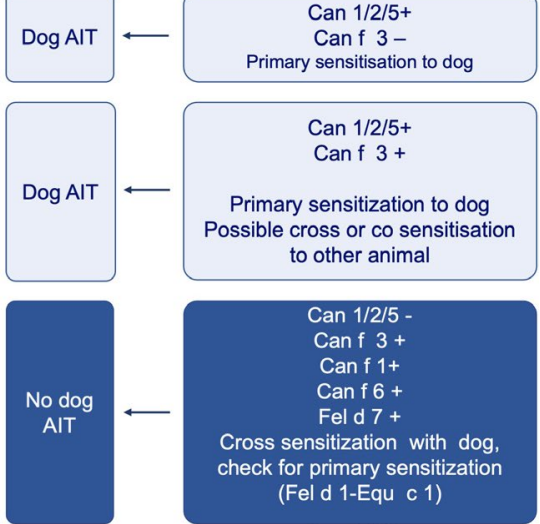
sensitization patterns, the lack of preparations with an adequate balance of major allergens is likely to explain this divergence. ${ }^{66,71,72}$

Further studies are needed to determine whether CRD could be used to identify patients who are most likely to respond to AIT specially in dog allergy.

Figure 5 summarizes decision-making algorithms in cat and dog allergy AIT selection.

\section{9 | Mite allergy}

Mite major allergens Der $p$ 1/Der $f 1$ and Der $p$ 2/Der $f$ sensitize the vast majority of mite-allergic patients, being the double sensitization to groups 1 and 2 strongly associated with asthma. ${ }^{32}$ Group 1 sensitization is more prevalent in children than in adults, suggesting an initiation role probably associated with its proteolytic properties. ${ }^{73}$ Recently, Der p 23 has been described to be associated with increased asthma risk. ${ }^{74}$ Other mite species, known as minor or storage mites, have a limited allergenic cross-reactivity with major mites. Interestingly, they display a significant cross-reactivity between them. Lep $d 2$ could be a good marker for minor storage allergy sensitization. ${ }^{75}$ Some patients exposed to high mite allergen levels, and sensitized to storage mites, develop anaphylactic reactions when exposed to foods contaminated with mites. ${ }^{76}$ Interestingly, these patients present NSAIDs intolerance, and recently, they have been described to present extensive oral mucosa barrier damage. ${ }^{8}$

A post hoc analysis of a study with AIT with $D$. pteronnysinus and $D$. farinae found no association between the clinical efficacy and sensitization to different mite allergens. ${ }^{77}$

Recently, the antibody response to a SCIT preparation for mite allergy has been examined at the molecular level in 24 mite-allergic patients. ${ }^{78}$ A protective IgG response has been observed for Der $\mathrm{p} 1$ and Der p 2 and, to a lesser extent, Der p 23. By contrast, patients did not develop a strong response to other mite allergens. Interestingly, a better clinical response to the AIT was observed in patients sensitized only to Der $\mathrm{p} 1$ and/or Der $\mathrm{p} 2$, when compared to patients with a broader IgE response. ${ }^{78}$ This suggests that the molecular profile of IgE sensitization may be useful in selecting patients that may benefit from subcutaneous AIT, ${ }^{78}$ as previously hypothesized. ${ }^{79}$ However no studies are available so far to ascertain whether this conclusion can be applied also to sublingual immunotherapy.

\subsection{0 | Hymenoptera venom allergy}

Apis mellifera or honeybee venom (HBV) is the best-characterized Hymenoptera venom due to the outstanding importance of this species as elicitor of venom allergy worldwide. Detailed genomic information and proteomic data of the pure venom are available. ${ }^{80-82}$ So far, 12 allergens of HBV are identified. ${ }^{83}$ Only two of them present substantial amounts in the venom, the major allergen phospholipase A2 (Api $m$ 1)(12\%) and the minor peptidic allergen melittin (Api $m$
4)(50\%). ${ }^{84}$ Despite their lower abundance in HBV compared to Api $\mathrm{m} 1$, a role as relevant major allergen was also confirmed for Api m 2 (hyaluronidase), Api m 3 (acid phosphatase), Api m 5 (dipeptidylpeptidase IV), and Api $\mathrm{m} 10$ (icarapin) with slgE reactivity in the range of $47.9 \%-52.2 \%, 49.6 \%-50 \%, 58.3 \%-61.7 \%$, and $61.8 \%-$ $72.2 \%$ of allergic patients' sera, respectively. ${ }^{85,86}$ For the other HBV allergens, less information about sensitization rates is available. ${ }^{87-89}$ Some HBV allergens have been identified as risk markers of more severe clinical phenotypes such as Api m 4 or of venom immunotherapy (VIT) failure such as Api $\mathrm{m} 10 .^{85,90}$

Prominent Vespula spp. (known as yellow jackets in the USA) allergens include phospholipase A1 (Ves $\vee 1$ ), hyaluronidase (Ves $\vee$ 2.0101), and antigen 5 (Ves $\vee 5$ ). ${ }^{91,92}$ A second hyaluronidase (Ves $\vee$ 2.0201), carrying an inactivation mutation in the active site of the enzyme, was identified, which seems to be the predominant isoform. ${ }^{93,94}$ Yellow jacket venom (YJV) also contains a dipeptidylpeptidase IV (Ves v 3) which shows high homology to HBV Api m $5 .{ }^{95}$ The sensitization rates of YJV-allergic patients to Ves $\vee 1$, Ves $\vee 3$, and Ves $v 5$ are $33.3 \%-54 \%,{ }^{96-101} 50 \%-62.8 \%,{ }^{95,96}$ and $84.5 \%-100 \%$, respectively. ${ }^{96,98,99,101,102,103}$ In contrast to HBV hyaluronidase Api m 2, which is a major allergen, the YJV homologue Ves $v 2$ seems to be of restricted relevance and sensitization was reported in 5\%-25\% of YJV-allergic patients. ${ }^{104}$

The allergen composition of Polistesdominula venom (PDV) is very similar to that of YJV and the most important allergens are phospholipase A1 (Pol d 1), dipeptidyl peptidase IV (Pol d 3), and antigen 5 (Pol d 5) with sensitization rates of $87 \%,{ }^{105} 66.7 \%,{ }^{106}$ and $69 \%-72 \%,{ }^{105}$ respectively.

CRD is helpful to discriminate between genuine double sensitization and cross-reactivity, allowing physicians to optimize patient selection for VIT. The potential of CRD becomes evident by the fact that HBV and YJV in addition to homologous allergens also contain differentiating marker allergens that are present in either HBV or YJV. This is not the case when differential diagnosis between Vespula spp. and Polistes spp. is required (Figure 6). CRD is also helpful in identifying patients with Hymenoptera venom-induced anaphylaxis having negative test results to whole venom extracts, as it can be the case in hymenoptera venom-allergic patients with mast cell disorders. ${ }^{107}$ Currently available allergens of honeybee and vespid venoms (see Table 2) allow a molecular diagnosis in the vast majority of patients, but not in $100 \%$ of them. Moreover, not all allergens are available for one assay system. New recombinant molecules are needed to improve the diagnosis of Polistesspp.-allergic patients, especially in the case of double-positivity to both Polistes spp. and Vespula spp. venom, in order to prevent unnecessary double VIT (Figure 7A). Although diagnostic sensitivity of the currently available allergen panel, particularly of HBV, is not $100 \%$, CRD has clearly improved discrimination of primary allergy and crossreactivity in YJV and HBV allergy, thus facilitating correct prescription of VIT. A suggested diagnostic algorithm to discriminate between HBV and YJV allergy using CRD is given in Figure 7B. Of note, the same algorithm using the corresponding PDV allergens can also be applied to discriminate between HBV and PDV allergy. 
FIGURE 6 Established discriminating and cross-reactive allergens of HBV, YJV, and PDV. While allergens are identified that enable a differentiation between HBV (Api m 1, Api m 3, Api m 4, and Api m 10) and YJV/PDV (Ves v 1/Pold 1 and Ves $\vee 5 /$ Pol d 5) sensitization, the so far known allergens of YJV and PDV exhibit crossreactivity

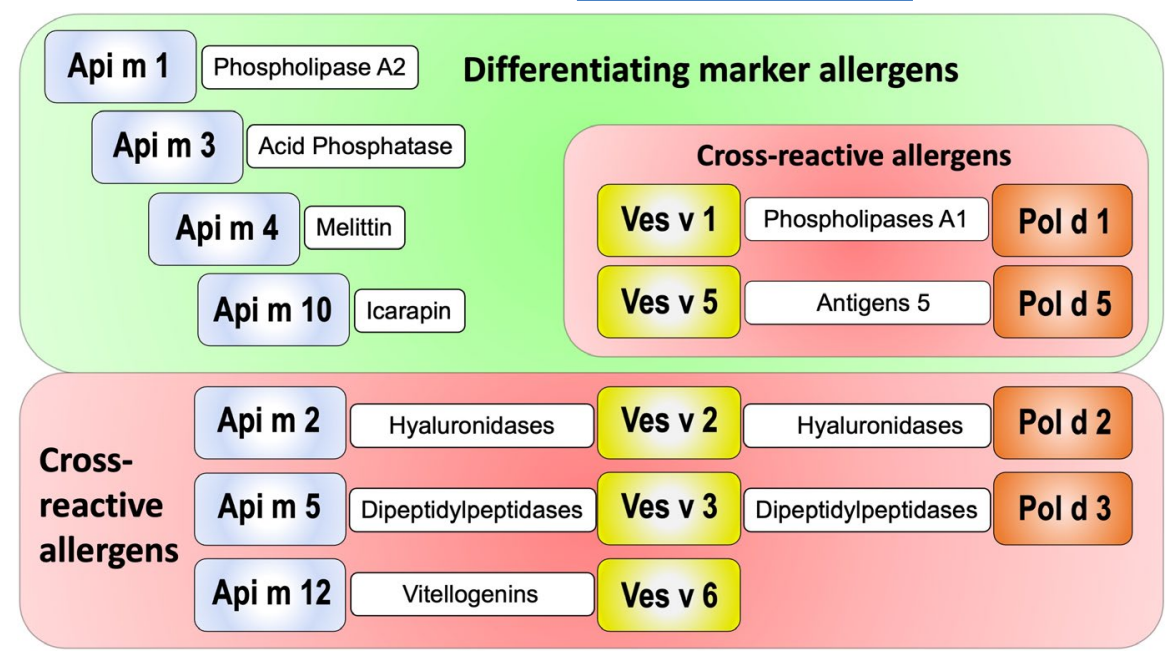

\subsection{1 | Peanut allergy}

A product for oral immunotherapy (OIT) to peanut has been recently licensed by FDA and EMA and will soon become widely available. ${ }^{108,109}$ Other products and routes of administration have been explored. ${ }^{110}$

Peanut allergic reactions can be triggered by storage proteins, such as Ara h 1, Ara h 2, Ara h 3, and Ara h 6, ${ }^{111}$ which have been associated to life-threatening symptoms, as well as by cross-reactive

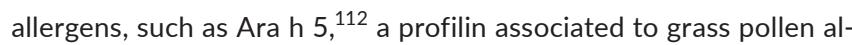
lergy, Ara $\mathrm{h}$ 8, belonging to PR10 family and associated to birch pollinosis and Ara h 9, a nsLTP, associated to the LTP syndrome discussed earlier(Table 1). Symptoms elicited by Ara $\mathrm{h} 5$ and Ara $\mathrm{h} 8$ are usually mild and limited to the oral cavity, the oropharynx, known as oral allergy syndrome. Ara h 2 slgE levels are associated to both severity and threshold of allergic reactions during oral provocation challenges, thus being a good biomarker for severity. ${ }^{113,114}$ In fact, Ara h 2 is the dominant $2 \mathrm{~S}$ globulin allergen. ${ }^{115}$ As storage proteins can trigger systemic food allergic reactions and anaphylaxis, peanut allergy with confirmed sensitization to seed storage proteins like Ara h 1 , Ara h 2 and Ara h 6 can constitute an indication for POIT to peanut. A slgE determination to a combination of the three allergens might constitute an economic diagnostic tool to assess anaphylaxis risk.

A summary of the decision process to select POIT is shown in Figure 8.

\section{3 | CRD AND AIT SAFETY}

In general, there are limited and heterogeneous evidences associating risk factors for adverse events during AIT and particular IgE sensitization profiles. The most studied model is grass pollen allergy. Adverse reactions with subcutaneous immunotherapy, both local and systemic, correlate with sensitization progression ( $\mathrm{Phl} p$ $1+5+12>\mathrm{Phl} \mathrm{p} 1+5>\mathrm{Phl} \mathrm{p} \mathrm{1/5).}{ }^{116}$ In sublingual AIT, adverse events were also related with highest slgE levels for Phl $\mathrm{p} 5$ or $\mathrm{Phl} p$ $1^{27}$ and with allergen sensitivity. ${ }^{117}$ Under extreme exposure to grass pollen, patients are frequently sensitized to profilin and present severe adverse reactions to foods caused by profilin. Interestingly, this type of these reactions to profilin-containing foods is similar to the infrequent reactions observed after application of SLIT. This rare phenotype constitutes therefore a unique model to understand disease severity and limits for AIT. Profilin severe reactors undergo extensive oral mucosa damage and present a unique systemic metabolic status that points to T-cell proliferation, sustained inflammation, and altered repair function. The fact that profilin-sensitized patients in Spain present an enhanced T-cell proliferation compared with similar patients from Denmark suggests that progression to severe phenotypes might be linked to an uncontrolled inflammation and T-cell proliferation. Interestingly, in the other severity models previously mentioned systemic barrier damage, uncontrolled effector cell response, and altered repair-associated biomarkers have been described. All cases might be explained by T-cell cross-reactive allergens: Profilin, Ole e 7 (a nsLTP from pollen) and Lep d 2 (a frequent food contaminant inducing reactions in patients with storage mite sensitization).

\section{4 | FUTURE DIRECTIONS}

Accurate diagnosis is essential before AIT can be considered. The routine use of molecular diagnosis for allergic diseases and AIT, theorized in the late 1990 s by Rudolf Valenta and colleagues, ${ }^{118}$ is relatively recent and is still evolving. Constraints include the perceived complexity of this diagnostic approach and the slightly higher costs of test execution. ${ }^{119-121}$ The recent development of a novel multiplex test containing both extracts and molecules relevant for pollen allergy (a sort of "molecular pollen test") may help doctors in the prescription of the appropriate AIT products. ${ }^{122}$ The development of algorithms and clinical decision support systems integrated into apps for smartphones ${ }^{123}$ will facilitate the clinical interpretation of the outcome of IgE molecular assays, as shown in a recent pilot experience (Arasi, $\mathrm{S}$ et al. Clin Exp Allergy, in press). Different estimates confirm that CRD has a significant impact on AIT formula selection. ${ }^{124-126}$ 
(A)
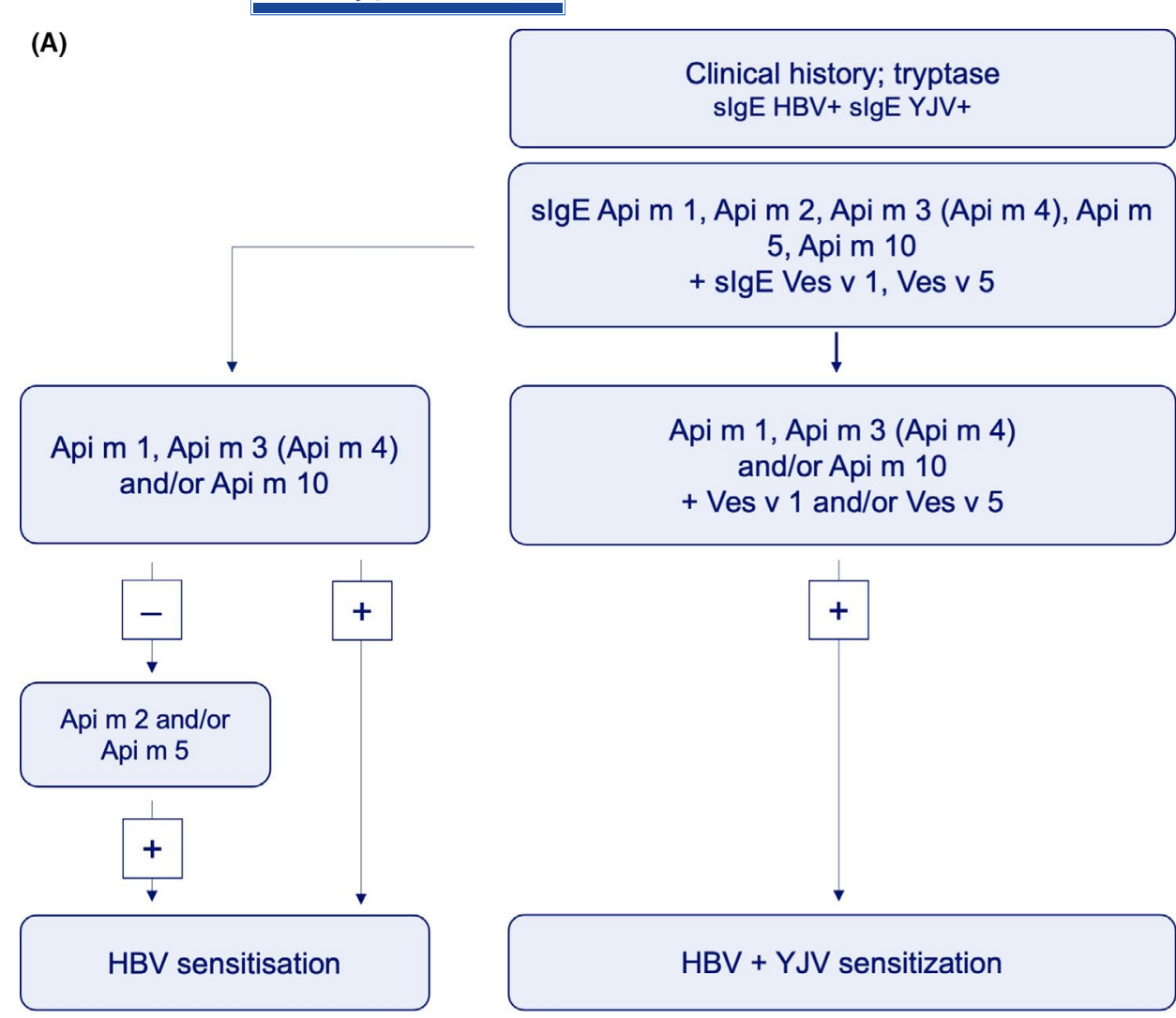

Api m 1, Api m 3 (Api m 4) and/or Api $m 10$

+ Ves $\vee 1$ and/or Ves v 5

Ves $v 1$ and/or Ves $\vee 5$

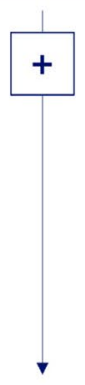

HBV + YJV sensitization

(B)

$$
\text { slgE YJV + slgE PDV + tryptase }
$$

slgE Ves v 1, Ves v $5+\operatorname{slg} E$ Pol d 5, (Pol d 1)

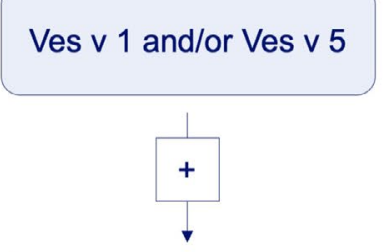

YJV sensitisation
Ves $v 1$ and/or Ves $v 5+$ Pol d 5 (and/or Pol d 1)

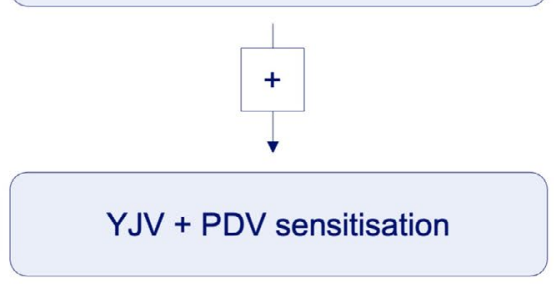

If only one culprit insect is likely, slgE levels to Ves $v 1$ and Pol $d 1$ + Ves $v 5$ and Pol d 5 might help for further discrimination

FIGURE 7 Diagnostic algorithm for in vitro diagnostics of (A) HBV and YJV allergy and (B) YJV and PDV allergy. The diagnostic algorithm shown in (A), with the corresponding PDV allergens Pol d 1 and Pol d 5, can also be used to discriminate between HBV and PDV allergy. In addition, to discriminate in the case of double-positive test results, CRD might also be useful in cases of negative results despite a convincing history of venom allergy or in cases of discrepancies between history, skin tests and venom extract-based testing. A plus indicates a positive and a minus a negative test result. Of note, the allergens Api $\mathrm{m} 4$ and Pol $\mathrm{d} 1$ (in brackets) are only available for selected multiplex slgE test platforms. *The allergens Api m 2 and Api m 5 are potentially cross-reactive with their homologues from YJV and PDV (not available for CRD) and, hence, a positive test results do not necessarily exclude allergy to vespid venom 


\section{Clinical history}

- Clear history of immediate allergic reactions OR positve oral food challenge to peanut

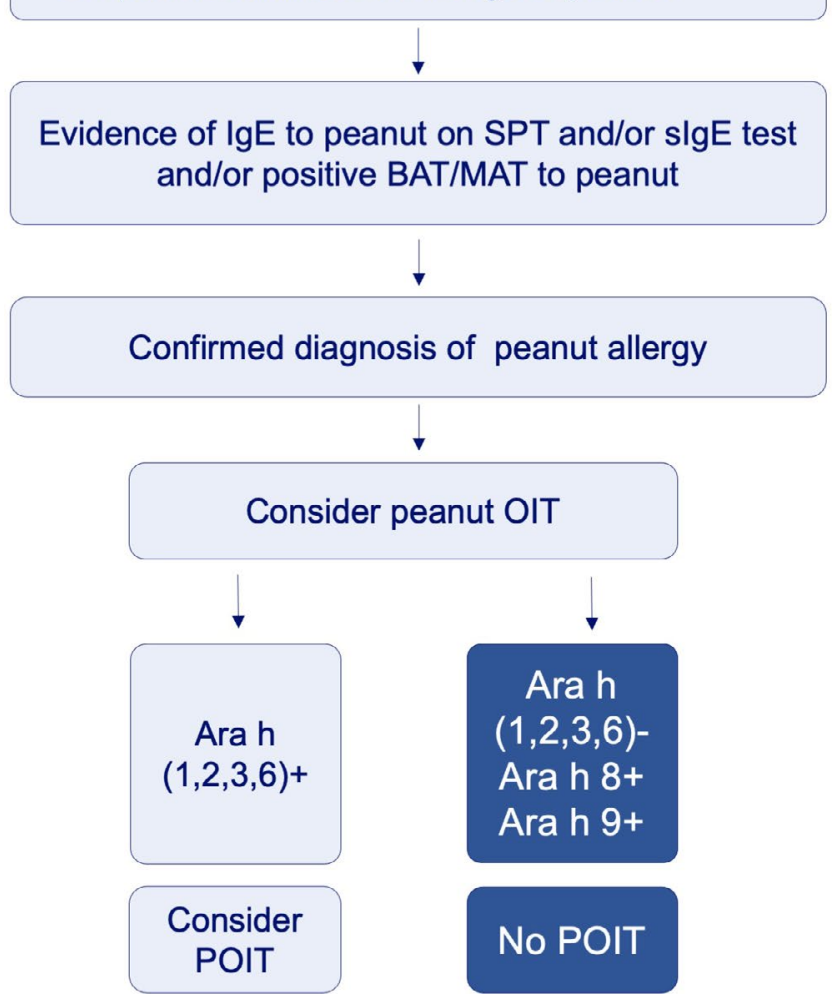

FIGURE 8 Diagnostic algorithm for in vitro diagnostics in peanut allergy oriented to make POIT decision. Discriminant analysis between storage allergens and cross-reactive to pollen and nsLTPs is proposed. Only patients with positive slgE to any of the Ara h 1 , 2, 3, 6 should be eligible for POIT

To rely on correctly standardized and clinically documented AIT products, as well as to understand their limitations, is as important as correctly diagnosing patients.

With an increasing number of new intervention possibilities, it is essential to optimize the use of AIT. ${ }^{58,127,128}$ Before considering AIT, patients should be sensitized to major allergens demonstrated by CRD and should be treated with one or two non-cross-reactive allergen preparations. In fact, even in complex exposure regions, most of the patients are sensitized to a limited number of allergens ${ }^{129}$ and thus potentially eligible for AIT. An adequate dose of allergen as defined in dose-finding clinical trials will guarantee an adequate safety/ efficacy balance. In the last years, an increasing number of allergen preparations developed with the highest pharmacological standards became available. This trend will continue and is expected to have a profound impact on AIT practice and to position it in the center of etiological management of allergic patients. In spite of correct diagnosis and the use of high-quality AIT products, a fraction of treated patients will not improve or will lose clinical benefit upon discontinuation after 3 years of intervention. ${ }^{13}$ The need to advance on personalized medicine approaches to predict intervention outcome and safety-and to monitor AIT effect-is imperative. ${ }^{130}$ System biology approaches are being explored to understand AIT response. ${ }^{5}$ These new approaches might help to understand biological effect kinetics and will provide new tools to evaluate new AIT product approaches. The desensitization of allergen-specific effector cells plays a determinant role for AIT effect during the first 2 years of intervention, while regulatory response, which is initiated shortly after AIT initiation, will only have a meaningful clinical benefit after at least $2 / 3$ years of administration. We need better formulas, including adjuvants and tolerogenic signaling molecules to reduce AIT duration. ${ }^{6}$ At the same time, understanding severe phenotypes is needed. Recent data obtained by system biology approaches identify T-cell proliferation, inadequate regulatory function, and collapse of repair homeostasis as main causes for non-response to intervention and evolution to severe phenotypes. ${ }^{7,8,9,131}$ There are new possibilities of using these systemic signatures to explore the value of new biologics to stabilize severe phenotypes allowing ulterior AIT intervention.

While CRD has proved the value for correct patient inclusion and AIT standardization, we cannot expect that it will be of great value to predict intervention outcome or safety. ${ }^{77}$ AIT is the only treatment in allergy able to modify the course of the disease, but for achieving this goal, the correct patients, with the correct product, with adequate treatment duration and compliance are needed. Even so, about $30 \%$ of patients will not benefit from this disease-modifying effect. This $70 \%$ effect, ${ }^{13}$ however, ranks at the top of successful pharmacological interventions. In 2003, Dr. Allen Roses, by that time vice president of the genetics division for GSK, commented that "The vast majority of medicines, more than 90 percent, only work in 30 or 50 percent of people." Since then, massive efforts on personalized medicine are progressively changing the landscape. AIT has the potential of being the reference intervention in allergy, but for that, we need a commitment and upgrade of its clinical practice. The use of molecular allergology for diagnostic purposes will rapidly evolve in the next years and will be more linked to the identification of patients phenotypes and endotypes for an improved therapeutic approach, which implies the opening of the era of "precision allergology". ${ }^{120,121}$ CRD, today, provides a good starting point.

\section{ACKNOWLEDGMENTS}

We thank Tomas Barker-Tejeda for the critical reading of the manuscript. Dr. Barber reports personal fees from ALK, AIMMUNE, grants from ALK, Allero therapeutics, outside the submitted work. Dr. Kleine-Tebbe reports personal fees from Allergen Online (Nebraska, USA), personal fees from Allergy Therapeutics, personal fees from Allergopharma, personal fees from ALK-Abelló, personal fees from AstraZeneca, personal fees from Bencard, personal fees from Dr. Pfleger, grants and personal fees from GSK, personal fees from HAL Allergy, personal fees from InfectoPharm, personal fees from LETI, grants and personal fees from Lofarma, grants and personal fees from Novartis, personal fees from Merck US, personal fees from Sanofi Genentech, grants and personal fees from Stallergenes-Greer, personal fees from Springer International Publishers, personal fees 
from ThermoFisher Scientific, personal fees from Thieme Publishers, Germany, non-financial support from American Academy Allergy Asthma and Immunology, non-financial support from European Academy of Allergy and Clinical Immunology, personal fees and non-financial support from German Society of Allergy and Clinical Immunology, non-financial support from WHO/IUIS Allergen nomenclature subcommittee, outside the submitted work. Dr. Matricardi reports personal fees from TPS, grants, personal fees and non-financial support from Thermo Fisher Scientific, grants and personal fees from Hycor, personal fees from Omron, grants from Stallergens, grants and personal fees from Euroimmun, outside the submitted work; Dr. Matricardi reports personal fees from TPS, grants, personal fees and non-financial support from Thermo Fisher Scientific, grants and personal fees from Hycor, grants and personal fees from Omron, grants from Stallergens, grants and personal fees from Euroimmun, outside the submitted work. Dr. Ollert reports personal fees from HycorDiagnostics, outside the submitted work. Dr. Sastr reports grants and personal fees from SANOFI, personal fees from GSK, personal fees from NOVARTIS, personal fees from ASTRA ZENECA, personal fees from MUNDIPHARMA, personal fees from FAES FARMA, outside the submitted work. All other authors have nothing to disclose.

\section{ORCID}

Domingo Barber (D) https://orcid.org/0000-0002-5488-5700

Araceli Diaz-Perales (DD https://orcid.org/0000-0002-1093-3627

Maria M. Escribese (D) https://orcid.org/0000-0001-5057-5150

Jörg Kleine-Tebbe (D) https://orcid.org/0000-0002-2862-7353

Paolo M. Matricardi (D) https://orcid.org/0000-0001-5485-0324

Markus Ollert (D) https://orcid.org/0000-0002-8055-0103

Alexandra F. Santos (D) https://orcid.org/0000-0002-7805-1436

Joaquin Sastre (D) https://orcid.org/0000-0003-4689-6837

\section{REFERENCES}

1. Bonertz A, Roberts GC, Hoefnagel M, et al. Challenges in the implementation of $\mathrm{EAACl}$ guidelines on allergen immunotherapy: $A$ global perspective on the regulation of allergen products. Allergy. 2018;73(1):64-76.

2. Bonertz A, Roberts G, Slater JE, et al. Allergen manufacturing and quality aspects for allergen immunotherapy in Europe and the United States: an analysis from the EAACI AIT Guidelines Project. Allergy. 2018;73(4):816-826.

3. van Ree R, Chapman MD, Ferreira F, et al. The CREATE project: development of certified reference materials for allergenic products and validation of methods for their quantification. Allergy. 2008;63(3):310-326.

4. Matricardi PM, Kleine-Tebbe J, Hoffmann HJ, et al. EAACI molecular allergology user's guide. Pediatr Allergy Immunol. 2016;27(Suppl 23):1-250.

5. Barker-Tejeda TC, Bazire R, Obeso D, et al. Exploring novel systemic biomarker approaches in grass-pollen sublingual immunotherapy using omics. Allergy. 2021;76:1199-1212.

6. Sokolowska M, Boonpiyathad T, Escribese MM, Barber D. Allergen-specific immunotherapy: power of adjuvants and novel predictive biomarkers. Allergy. 2019;74(11):2061-2063.

7. Barber D, Villasenor A, Escribese MM. Metabolomics strategies to discover new biomarkers associated to severe allergic phenotypes. Asia Pac Allergy. 2019;9(4):e37.
8. Sanchez-Solares J, Delgado-Dolset MI, Mera-Berriatua L, et al. Respiratory allergies with no associated food allergy disrupt oral mucosa integrity. Allergy. 2019;74(11):2261-2265.

9. Obeso D, Mera-Berriatua L, Rodríguez-Coira J, et al. Multi-omics analysis points to altered platelet functions in severe foodassociated respiratory allergy. Allergy. 2018;73(11):2137-2149.

10. Rosace D, Gomez-Casado C, Fernandez P, et al. Profilin-mediated food-induced allergic reactions are associated with oral epithelial remodeling. J Allergy Clin Immunol. 2019;143(2):681-690.e681.

11. Eguiluz-Gracia I, Tay TR, Hew M, et al. Recent developments and highlights in biomarkers in allergic diseases and asthma. Allergy. 2018;73(12):2290-2305.

12. Barber D, Rico P, Blanco C, Fernandez-Rivas M, Ibanez MD, Escribese MM. GRAZAX(R): a sublingual immunotherapy vaccine for Hay fever treatment: from concept to commercialization. Hum Vaccin Immunother. 2019;15(12):2887-2895.

13. Varona R, Ramos T, Escribese MM, et al. Persistent regulatory Tcell response 2 years after 3 years of grass tablet SLIT: Links to reduced eosinophil counts, slgE levels, and clinical benefit. Allergy. 2019;74(2):349-360.

14. Suarez-Fueyo A, Ramos T, Galan A, et al. Grass tablet sublingual immunotherapy downregulates the $\mathrm{TH} 2$ cytokine response followed by regulatory T-cell generation. J Allergy Clin Immunol. 2014;133(1):130-138.e131-132.

15. van de Veen W, Akdis $M$. Tolerance mechanisms of allergen immunotherapy. Allergy. 2020;75(5):1017-1018.

16. Satitsuksanoa $P$, van de Veen W, Akdis M. B-cell responses in allergen immunotherapy. Curr Opin Allergy Clin Immunol. 2019;19(6):632-639.

17. Jansen K, Cevhertas L, Ma S, Satitsuksanoa P, Akdis M, van de Veen W. Regulatory B cells, A to Z. Allergy. 2021. https://doi. org/10.1111/all.14763. Online ahead of print.

18. Ma S, Satitsuksanoa P, Jansen K, Cevhertas L, van de Veen W, Akdis M. B regulatory cells in allergy. Immunol Rev. 2021;299(1):10-30.

19. Eljaszewicz A, Ruchti F, Radzikowska $U$, et al. Trained immunity and tolerance in innate lymphoid cells, monocytes, and dendritic cells during allergen-specific immunotherapy. J Allergy Clin Immunol. 2021;147s:1865-1877.

20. Ansotegui JI, Melioli F, Canonica GW, et al. A WAO - ARIA - GA(2) LEN consensus document on molecular-based allergy diagnosis (PAMD@): Update 2020. World Allergy Organ J. 2020;13(2):100091.

21. Goodman RE, Breiteneder H. The WHO/IUIS allergen nomenclature. Allergy. 2019;74(3):429-431.

22. Costa J, Bavaro SL, Benedé S, et al. Are physicochemical properties shaping the allergenic potency of plant allergens? Clin Rev Allergy Immunol. 2020. https://doi.org/10.1007/s12016-02008810-9. Online ahead of print.

23. Costa J, Villa C, Verhoeckx K, et al. Are physicochemical properties shaping the allergenic potency of animal allergens? Clin Rev Allergy Immunol. 2021. https://doi.org/10.1007/s12016-020-08826-1. Online ahead of print.

24. Kaul S, Zimmer J, Dehus O, et al. Validation of ELISA methods for quantification of the major birch allergen Bet $v 1$ (BSP090). Pharmeur Bio Sci Notes. 2017;2017:69-87.

25. Kaul S, Zimmer J, Dehus O, et al. Standardization of allergen products: 3. Validation of candidate European Pharmacopoeia standard methods for quantification of major birch allergen Bet $v 1$. Allergy. 2016;71(10):1414-1424.

26. Vieths S, Barber D, Chapman M, et al. Establishment of recombinant major allergens Bet $v 1$ and $\mathrm{Phl} p 5 \mathrm{a}$ as Ph. Eur. reference standards and validation of ELISA methods for their measurement. Results from feasibility studies. Pharmeur Bio Sci Notes. 2012;2012:118-134.

27. Nolte M, Barber D, Maloney J, et al. Timothy specific IgE levels are associated with efficacy and safety of timothy grass 
sublingual immunotherapy tablet. Ann Allergy Asthma Immunol. 2015;115(6):509-515.e502.

28. Duffort O, Palomares O, Lombardero M, et al. Variability of Ole e 9 allergen in olive pollen extracts: relevance of minor allergens in immunotherapy treatments. Int Arch Allergy Immunol. 2006;140(2):131-138.

29. Barber D, Moreno C, Ledesma A, et al. Degree of olive pollen exposure and sensitization patterns. Clinical implications. J Investig Allergol Clin Immunol. 2007;17(Suppl 1):11-16.

30. Hatzler L, Panetta V, Lau S, et al. Molecular spreading and predictive value of preclinical IgE response to Phleum pratense in children with hay fever. J Allergy Clin Immunol. 2012;130(4):894-901.e895.

31. Posa D, Perna S, Resch Y, et al. Evolution and predictive value of IgE responses toward a comprehensive panel of house dust mite allergens during the first 2 decades of life. J Allergy Clin Immunol. 2017;139(2):541-549.e548.

32. Custovic A, Sonntag HJ, Buchan IE, Belgrave D, Simpson A, Prosperi MCF. Evolution pathways of IgE responses to grass and mite allergens throughout childhood. J Allergy Clin Immunol. 2015;136(6):1645-1652.e1648.

33. Matricardi PM. Allergen-specific immunoprophylaxis: toward secondary prevention of allergic rhinitis? Pediatr Allergy Immunol. 2014;25(1):15-18.

34. Lund G, Brand S, Ramos T, et al. Strong and frequent T-cell responses to the minor allergen $\mathrm{Phl} \mathrm{p} 12$ in Spanish patients IgEsensitized to Profilins. Allergy. 2018;73(5):1013-1021.

35. Barber D, de la Torre F, Feo F, et al. Understanding patient sensitization profiles in complex pollen areas: a molecular epidemiological study. Allergy. 2008;63(11):1550-1558.

36. Barber D, de la Torre F, Lombardero M, et al. Component-resolved diagnosis of pollen allergy based on skin testing with profilin, polcalcin and lipid transfer protein pan-allergens. Clin Exp Allergy. 2009;39(11):1764-1773.

37. Cipriani F, Mastrorilli C, Tripodi S, et al. Diagnostic relevance of IgE sensitization profiles to eight recombinant Phleum pratense molecules. Allergy. 2018;73(3):673-682.

38. Hernández-Ramírez G, Pazos-Castro D, Gómez Torrijos E, et al. Group 1 allergens, transported by mold spores, induce asthma exacerbation in a mouse model. Allergy. 2020;75(9):2388-2391.

39. Asero R, Monsalve R, Barber D. Profilin sensitization detected in the office by skin prick test: a study of prevalence and clinical relevance of profilin as a plant food allergen. Clin Exp Allergy. 2008;38(6):1033-1037.

40. Ruiz-Garcia M, Garcia Del Potro M, Fernandez-Nieto M, Barber D, Jimeno-Nogales L, Sastre J. Profilin: a relevant aeroallergen? J Allergy Clin Immunol. 2011;128(2):416-418.

41. Alvarado MI, Jimeno L, De La Torre F, et al. Profilin as a severe food allergen in allergic patients overexposed to grass pollen. Allergy. 2014;69(12):1610-1616.

42. Rodríguez del Río P, Díaz-Perales A, Sánchez-García S, et al. Profilin, a change in the paradigm. J Investig Allergol Clin Immunol. 2018;28(1):1-12

43. Matricardi PM. IgE to cross-reactive carbohydrate determinants: Origins, functions, and confounding role in $\mathrm{nPhl} p$ 4-IgE assays. $J$ Allergy Clin Immunol. 2020;145(6):1554-1555.

44. Salcedo G, Sanchez-Monge R, Barber D, Diaz-Perales A. Plant nonspecific lipid transfer proteins: an interface between plant defence and human allergy. Biochim Biophys Acta. 2007;1771(6):781-791.

45. Skypala IJ, Bartra J, Ebo DG, et al. The diagnosis and management of allergic reactions in patients sensitized to non-specific Lipid Transfer Proteins. Allergy. 2021. https://doi.org/10.1111/ all.14797. Online ahead of print

46. Fernández-Rivas $M$, Garrido Fernández $S$, Nadal JA, et al. Randomized double-blind, placebo-controlled trial of sublingual immunotherapy with a Pru p 3 quantified peach extract. Allergy. 2009;64(6):876-883.
47. Gomez F, Bogas G, Gonzalez M, et al. The clinical and immunological effects of Pru p 3 sublingual immunotherapy on peach and peanut allergy in patients with systemic reactions. Clin Exp Allergy. 2017;47(3):339-350.

48. Palomares F, Gomez F, Bogas G, et al. Immunological changes induced in peach allergy patients with systemic reactions by Pru $\mathrm{p} 3$ sublingual immunotherapy. Mol Nutr Food Res. 2018;62(3):1700669.

49. Biedermann T, Winther L, Till SJ, Panzner P, Knulst A, Valovirta E. Birch pollen allergy in Europe. Allergy. 2019;74(7):1237-1248.

50. Biedermann T, Kuna P, Panzner P, et al. The SQ tree SLIT-tablet is highly effective and well tolerated: Results from a randomized, double-blind, placebo-controlled phase III trial. J Allergy Clin Immunol. 2019;143(3):1058-1066.e1056.

51. Nolte $\mathrm{H}$, Waserman $\mathrm{S}$, Ellis AK, Biedermann T, Wurtzen PA Treatment effect of the tree pollen SLIT-tablet on allergic rhinoconjunctivitis during oak pollen season. J Allergy Clin Immunol Pract. 2021;9:1871-1878.

52. Würtzen PA, Grønager PM, Lund G, et al. Simplified AIT for allergy to several tree pollens-Arguments from the immune outcome analyses following treatment with SQ tree SLIT-tablet. Clin Exp Allergy. 2021;51(2):284-295

53. van der Valk JPM, Nagl B, van Wljk RG, Bohle B, de Jong NW. The effect of birch pollen immunotherapy on apple and rMal d 1 challenges in adults with apple allergy. Nutrients. 2020;12(2):519.

54. Till SJ, Stage BS, Skypala I, Biedermann T. Potential treatment effect of the SQ tree SLIT-tablet on pollen food syndrome caused by apple. Allergy. 2020;75(8):2059-2061.

55. Sanchez Acosta G, Kinaciyan T, Kitzmuller C, Mobs C, Pfutzner W, Bohle B. IgE-blocking antibodies following SLIT with recombinant Mal d 1 accord with improved apple allergy. J Allergy Clin Immunol. 2020;146(4):894-900.e892.

56. Okamoto Y, Okubo K, Yonekura S, et al. Efficacy and safety of sublingual immunotherapy for two seasons in patients with Japanese cedar pollinosis. Int Arch Allergy Immunol. 2015;166(3):177-188.

57. Gotoh M, Yonekura S, Imai T, et al. Long-term efficacy and dosefinding trial of Japanese cedar pollen sublingual immunotherapy tablet. J Allergy Clin Immunol Pract. 2019;7(4):1287-1297.e1288.

58. Alvaro-Lozano $\mathrm{M}$, Akdis CA, Akdis $\mathrm{M}$, et al. EAACl allergen immunotherapy user's guide. Pediatr Allergy Immunol. 2020;31(Suppl 25):1-101

59. Christensen LH, Ipsen $\mathrm{H}$, Nolte $\mathrm{H}$, et al. Short ragweeds is highly cross-reactive with other ragweeds. Ann Allergy Asthma Immunol. 2015;115(6):490-495.e491.

60. Creticos PS, Maloney J, Bernstein DI, et al. Randomized controlled trial of a ragweed allergy immunotherapy tablet in North American and European adults. J Allergy Clin Immunol. 2013;131(5):13421349.e1346.

61. Nolte H, Bernstein DI, Nelson HS, Ellis AK, Kleine-Tebbe J, Lu S. Efficacy and safety of ragweed SLIT-tablet in children with allergic rhinoconjunctivitis in a randomized, placebo-controlled trial. $J$ Allergy Clin Immunol Pract. 2020;8(7):2322-2331.e2325.

62. Uriarte SA, Sastre J. Clinical relevance of molecular diagnosis in pet allergy. Allergy. 2016;71(7):1066-1068.

63. Ukleja-Sokołowska N, Gawrońska-Ukleja E, Żbikowska-Gotz M, et al. Analysis of feline and canine allergen components in patients sensitized to pets. Allergy Asthma Clin Immunol. 2016;12:61.

64. Nordlund B, Konradsen JR, Kull I, et al. IgE antibodies to animalderived lipocalin, kallikrein and secretoglobin are markers of bronchial inflammation in severe childhood asthma. Allergy. 2012;67(5):661-669.

65. Schoos AM, Nwaru BI, Borres MP. Component-resolved diagnostics in pet allergy: current perspectives and future directions. $J$ Allergy Clin Immunol. 2021;147(4):1164-1173.

66. Uriarte SA, Gronlund H, Wintersand A, Bronge J, Sastre J. Clinical and immunologic changes due to subcutaneous immunotherapy with cat and dog extracts using an ultrarush up-dosing phase: a 
real-life study. J Investig Allergol Clin Immunol. 2020;32. Online ahead of print.

67. Asarnoj A, Hamsten C, Waden K, et al. Sensitization to cat and dog allergen molecules in childhood and prediction of symptoms of cat and dog allergy in adolescence: A BAMSE/MeDALL study. J Allergy Clin Immunol. 2016;137(3):813-821.e817.

68. Nanda A, O'Connor M, Anand M, et al. Dose dependence and time course of the immunologic response to administration of standardized cat allergen extract. J Allergy Clin Immunol. 2004;114(6):1339-1344.

69. Wintersand A, Asplund K, Binnmyr J, et al. Allergens in dog extracts: Implication for diagnosis and treatment. Allergy. 2019;74(8):1472-1479.

70. Lent AM, Harbeck R, Strand M, et al. Immunologic response to administration of standardized dog allergen extract at differing doses. J Allergy Clin Immunol. 2006;118(6):1249-1256.

71. Uriarte SA, Sastre J. Subcutaneous immunotherapy with highdose cat and dog extracts: a real-life study. J Investig Allergol Clin Immunol. 2020;30(3):169-174.

72. Uriarte S, Sastre J. Safety of an Ultrarush (4 hours) subcutaneous immunotherapy schedule with cat and dog extracts using an infusion pump. J Investig Allergol Clin Immunol. 2018;28(6):430-432.

73. Barber D, Arias J, Boquete $\mathrm{M}$, et al. Analysis of mite allergic patients in a diverse territory by improved diagnostic tools. Clin Exp Allergy. 2012;42(7):1129-1138.

74. Celi G, Brusca I, Scala E, et al. House dust mite allergy in ItalyDiagnostic and clinical relevance of Der p 23 (and of minor allergens): a real-life, multicenter study. Allergy. 2019;74(9):1787-1789.

75. Arias-Irigoyen J, Lombardero M, Arteaga C, Carpizo JA, Barber D. Limited IgE cross-reactivity between Dermatophagoides pteronyssinus and Glycyphagus domesticus in patients naturally exposed to both mite species. J Allergy Clin Immunol. 2007;120(1):98-104.

76. Blanco C, Quiralte J, Castillo R, et al. Anaphylaxis after ingestion of wheat flour contaminated with mites. J Allergy Clin Immunol. 1997:99(3):308-313.

77. Arroabarren E, Echechipia S, Galbete A, Lizaso MT, Olaguibel JM, Tabar Al. Association between component-resolved diagnosis of house dust mite allergy and efficacy and safety of specific immunotherapy. J Investig Allergol Clin Immunol. 2019;29(2):164-167.

78. Rodríguez-Domínguez A, Berings M, Rohrbach A, et al. Molecular profiling of allergen-specific antibody responses may enhance success of specific immunotherapy. J Allergy Clin Immunol. 2020;146(5):1097-1108.

79. Tripodi S, Frediani T, Lucarelli S, et al. Molecular profiles of IgE to Phleum pratense in children with grass pollen allergy: implications for specific immunotherapy. J Allergy Clin Immunol. 2012;129(3):834-839.e838.

80. Weinstock GM, Robinson GE, Gibbs RA, et al. Insights into socia insects from the genome of the honeybee Apis mellifera. Nature. 2006;443(7114):931-949.

81. Peiren N, Vanrobaeys F, de Graaf DC, Devreese B, Van Beeumen J, Jacobs FJ. The protein composition of honeybee venom reconsidered by a proteomic approach. Biochim Biophys Acta. 2005;1752(1):1-5.

82. Van Vaerenbergh M, Debyser G, Devreese B, de Graaf DC. Exploring the hidden honeybee (Apis mellifera) venom proteome by integrating a combinatorial peptide ligand library approach with FTMS. J Proteomics. 2014;99:169-178.

83. Radauer C, Nandy A, Ferreira F, et al. Update of the WHO/IUIS Allergen Nomenclature Database based on analysis of allergen sequences. Allergy. 2014;69(4):413-419.

84. Spillner E, Blank S, Jakob T. Hymenoptera allergens: from venom to "venome". Front Immunol. 2014;5:77.

85. Frick M, Fischer J, Helbling A, et al. Predominant Api $\mathrm{m} 10$ sensitization as risk factor for treatment failure in honey bee venom immunotherapy. J Allergy Clin Immunol. 2016;138(6):1663-1671.e1669.
86. Kohler J, Blank S, Muller S, et al. Component resolution reveals additional major allergens in patients with honeybee venom allergy. J Allergy Clin Immunol. 2014;133(5):1383-1389, 1389.e1381-1386.

87. Blank S, Bantleon FI, Mclntyre M, Ollert M, Spillner E. The major royal jelly proteins 8 and 9 (Api $m$ 11) are glycosylated components of Apis mellifera venom with allergenic potential beyond carbohydrate-based reactivity. Clin Exp Allergy. 2012;42(6):976-985

88. Blank S, Seismann H, Mclntyre M, et al. Vitellogenins are new high molecular weight components and allergens (Api m 12 and Ves $\vee 6$ ) of Apis mellifera and Vespula vulgaris venom. PLoS One. 2013;8(4):e62009.

89. Michel $\mathrm{Y}$, Mclntyre $\mathrm{M}$, Ginglinger $\mathrm{H}$, et al. The putative serine protease inhibitor Api $\mathrm{m} 6$ from Apis mellifera venom: recombinant and structural evaluation. J Investig Allergol Clin Immunol. 2012;22(7):476-484.

90. Ruiz B, Serrano P, Verdu M, Moreno C. Sensitization to Api m 1, Api $m$ 2, and Api $m$ 4: association with safety of bee venom immunotherapy. Ann Allergy Asthma Immunol. 2015;114(4):350-352.

91. King TP, Spangfort MD. Structure and biology of stinging insect venom allergens. Int Arch Allergy Immunol. 2000;123(2):99-106.

92. Muller UR. Recombinant Hymenoptera venom allergens. Allergy. 2002;57(7):570-576

93. Kolarich D, Leonard R, Hemmer W, Altmann F. The N-glycans of yellow jacket venom hyaluronidases and the protein sequence of its major isoform in Vespula vulgaris. FEBS J. 2005;272(20):5182-5190.

94. Seismann H, Blank S, Braren I, et al. Dissecting cross-reactivity in hymenoptera venom allergy by circumvention of alpha-1,3-core fucosylation. Mol Immunol. 2010;47(4):799-808.

95. Blank S, Seismann H, Bockisch B, et al. Identification, recombinant expression, and characterization of the $100 \mathrm{kDa}$ high molecular weight Hymenoptera venom allergens Api m 5 and Ves v 3. J Immunol. 2010;184(9):5403-5413.

96. Cifuentes L, Vosseler S, Blank S, et al. Identification of Hymenoptera venom-allergic patients with negative specific IgE to venom extract by using recombinant allergens. J Allergy Clin Immunol. 2014;133(3):909-910.

97. Ebo DG, Faber M, Sabato V, Leysen J, Bridts CH, De Clerck LS. Component-resolved diagnosis of wasp (yellow jacket) venom allergy. Clin Exp Allergy. 2013;43(2):255-261.

98. Korošec P, Valenta R, Mittermann I, et al. High sensitivity of CAPFEIA rVes $v 5$ and $r$ Ves $v 1$ for diagnosis of Vespula venom allergy. J Allergy Clin Immunol. 2012;129(5):1406-1408.

99. Mittermann I, Zidarn M, Silar M, et al. Recombinant allergenbased IgE testing to distinguish bee and wasp allergy. J Allergy Clin Immunol. 2010;125(6):1300-1307.e1303.

100. Seismann H, Blank S, Cifuentes L, et al. Recombinant phospholipase $A 1$ ( Ves $\vee 1$ ) from yellow jacket venom for improved diagnosis of hymenoptera venom hypersensitivity. Clin Mol Allergy. 2010;8:7.

101. Vos B, Kohler J, Muller S, Stretz E, Rueff F, Jakob T. Spiking venom with rVes $v 5$ improves sensitivity of IgE detection in patients with allergy to Vespula venom. J Allergy Clin Immunol. 2013;131(4):12251227, 1227.e1221.

102. Hofmann SC, Pfender N, Weckesser S, Huss-Marp J, Jakob T. Added value of IgE detection to rApi $m 1$ and rVes $\vee 5$ in patients with Hymenoptera venom allergy. J Allergy Clin Immunol. 2011;127(1):265-267

103. Muller UR, Johansen N, Petersen AB, Fromberg-Nielsen J, Haeberli G. Hymenoptera venom allergy: analysis of double positivity to honey bee and Vespula venom by estimation of IgE antibodies to species-specific major allergens Api m1 and Ves v5. Allergy. 2009;64(4):543-548.

104. Jin C, Focke M, Leonard R, Jarisch R, Altmann F, Hemmer W. Reassessing the role of hyaluronidase in yellow jacket venom allergy. J Allergy Clin Immunol. 2010;125(1):184-190.e181. 
105. Monsalve RI, Vega A, Marqués L, et al. Component-resolved diagnosis of vespid venom-allergic individuals: phospholipases and antigen 5 s are necessary to identify Vespula or Polistes sensitization. Allergy. 2012;67(4):528-536.

106. Schiener M, Hilger C, Eberlein B, et al. The high molecular weight dipeptidyl peptidase IV Pol d 3 is a major allergen of Polistes dominula venom. Sci Rep. 2018;8(1):1318.

107. Michel J, Brockow K, Darsow U, et al. Added sensitivity of component-resolved diagnosis in hymenoptera venom-allergic patients with elevated serum tryptase and/or mastocytosis. Allergy. 2016;71(5):651-660.

108. Vickery BP, Vereda A, Casale TB, et al. AR101 oral immunotherapy for peanut allergy. N Engl J Med. 2018;379(21):1991-2001.

109. O'B Hourihane J, Beyer K, Abbas A, et al. Efficacy and safety of oral immunotherapy with AR101 in European children with a peanut allergy (ARTEMIS): a multicentre, double-blind, randomised, placebo-controlled phase 3 trial. Lancet Child Adolesc Health. 2020;4(10):728-739.

110. Wang J. Advances in the management of peanut allergy (oral immunotherapy and epicutaneous immunotherapy). Allergy Asthma Proc. 2020;41(1):5-9.

111. Foong RX, Dantzer JA, Wood RA, Santos AF. Improving diagnostic accuracy in food allergy. J Allergy Clin Immunol Pract. 2021;9(1):71-80.

112. Santos AF, Barbosa-Morais NL, Hurlburt BK, et al. IgE to epitopes of Ara h 2 enhance the diagnostic accuracy of Ara h 2-specific IgE. Allergy. 2020;75:2309-2318.

113. Santos AF, Du Toit G, O'Rourke C, et al. Biomarkers of severity and threshold of allergic reactions during oral peanut challenges. $J$ Allergy Clin Immunol. 2020;146(2):344-355.

114. Santos AF. Food allergy severity prediction: quite a way to go yet? Expert Rev Clin Immunol. 2020;16(6):543-546.

115. Hemmings O, Du Toit G, Radulovic S, Lack G, Santos AF. Ara h 2 is the dominant peanut allergen despite similarities with Ara h 6. J Allergy Clin Immunol. 2020;146(3):621-630.e625.

116. Sastre J, Rodriguez F, Campo P, Laffond E, Marin A, Alonso MD. Adverse reactions to immunotherapy are associated with different patterns of sensitization to grass allergens. Allergy. 2015;70(5):598-600.

117. Calderon MA, Simons FE, Malling HJ, Lockey RF, Moingeon P, Demoly P. Sublingual allergen immunotherapy: mode of action and its relationship with the safety profile. Allergy. 2012;67(3):302-311.

118. Valenta R, Lidholm J, Niederberger V, Hayek B, Kraft D, Gronlund $H$. The recombinant allergen-based concept of componentresolved diagnostics and immunotherapy (CRD and CRIT). Clin Exp Allergy. 1999;29(7):896-904.

119. Matricardi PM, Dramburg S, Potapova E, Skevaki C, Renz H. Molecular diagnosis for allergen immunotherapy. J Allergy Clin Immunol. 2019;143(3):831-843.

120. Canonica GW, Bachert C, Hellings P, et al. Allergen Immunotherapy (AIT): a prototype of Precision Medicine. World Allergy Organ J. 2015;8(1):31.
121. Matricardi PM, Kleine-Tebbe J. Molecular allergology between precision medicine and the choosing wisely initiative. Clin Exp Allergy. 2016;46(5):664-667.

122. Di Fraia M, Arasi S, Castelli S, et al. A new molecular multiplex IgE assay for the diagnosis of pollen allergy in Mediterranean countries: a validation study. Clin Exp Allergy. 2019;49(3):341-349.

123. Matricardi PM, Potapova E, Forchert L, Dramburg S, Tripodi S. Digital allergology: towards a clinical decision support system for allergen immunotherapy. Pediatr Allergy Immunol. 2020;31(Suppl 24):61-64.

124. Kleine-Tebbe J, Matricardi PM, Hamilton RG. Allergy work-up including component-resolved diagnosis: how to make allergenspecific immunotherapy more specific. Immunol Allergy Clin North Am. 2016;36(1):191-203

125. Sastre J, Landivar ME, Ruiz-Garcia M, Andregnette-Rosigno MV, Mahillo I. How molecular diagnosis can change allergen-specific immunotherapy prescription in a complex pollen area. Allergy. 2012;67(5):709-711.

126. Sastre J, Sastre-lbanez M. Molecular diagnosis and immunotherapy. Curr Opin Allergy Clin Immunol. 2016;16(6):565-570.

127. Agache I, Lau S, Akdis CA, et al. EAACl guidelines on allergen immunotherapy: house dust mite-driven allergic asthma. Allergy. 2019;74(5):855-873.

128. Pajno GB, Fernandez-Rivas M, Arasi S, et al. EAACl Guidelines on allergen immunotherapy: IgE-mediated food allergy. Allergy. 2018;73(4):799-815.

129. Barber D, Diaz-Perales A, Villalba M, Chivato T. Challenges for allergy diagnosis in regions with complex pollen exposures. Curr Allergy Asthma Rep. 2015;15(2):496.

130. Shamji MH, Akdis CA, Barber D, et al. EAACl research and outreach committee: improving standards and facilitating global collaboration through a research excellence network. Allergy. 2020;75(8):1899-1901.

131. Gomez-Casado C, Villasenor A, Rodriguez-Nogales A, Bueno JL, Barber D, Escribese MM. Understanding platelets in infectious and allergic lung diseases. Int J Mol Sci. 2019;20(7):1730.

132. Blank S, Bilo MB, Ollert M. Component-resolved diagnostics to direct in venom immunotherapy: important steps towards precision medicine. Clin Exp Allergy. 2018;48(4):354-364.

How to cite this article: Barber D, Diaz-Perales A, Escribese $\mathrm{MM}$, et al. Molecular allergology and its impact in specific allergy diagnosis and therapy. Allergy. 2021;00:1-17. https:// doi.org/10.1111/all.14969 